ESAIM: M2AN

Vol. 40, No 4, 2006, pp. 765-784

DOI: $10.1051 / \mathrm{m} 2 \mathrm{an}: 2006029$
ESAIM: Mathematical Modelling and Numerical Analysis

www.edpsciences.org/m2an

\title{
NEW UNILATERAL PROBLEMS IN STRATIGRAPHY
}

\author{
Stanislav N. Antontsev ${ }^{1}$, Gérard Gagneux ${ }^{2}$, Robert Luce ${ }^{2}$ and Guy Vallet ${ }^{2}$
}

\begin{abstract}
This work deals with the study of some stratigraphic models for the formation of geological basins under a maximal erosion rate constrain. It leads to introduce differential inclusions of degenerated hyperbolic-parabolic type $0 \in \partial_{t} u-\operatorname{div}\left\{H\left(\partial_{t} u+E\right) \nabla u\right\}$, where $H$ is the maximal monotonous graph of the Heaviside function and $E$ is a given non-negative function. Firstly, we present the new and realistic models and an original mathematical formulation, taking into account the weather-limited rate constraint in the conservation law, with a unilateral constraint on the outflow boundary. Then, we give a study of the $1-D$ case with numerical illustrations.
\end{abstract}

Mathematics Subject Classification. 35K65, 35L80, 35Q35.

Received: October 24, 2005. Revised: March 20, 2006.

\section{INTRODUCTION}

In this paper we are interested in the mathematical analysis of general models arising in geological basin formation. The initial model has been developed by the Institut Français du Pétrole (IFP) and it takes into account sedimentation, transport and accumulation, erosion phenomena at large scales in time and space. The main feature of these models is characterized by a constraint on the time-derivative of the solution. This constraint leads us to consider an original class of conservation laws, a priori parabolic, revealing some hyperbolic behaviour because of a diffusive coefficient that depends on the time-derivative of the solution.

A more precise description of these models have been exposed, on the one hand, for the multilithological case, by Eymard et al. [9] and Gervais et al. [15]; on the second hand, by Antontsev et al. [3,4], Gagneux et al. [10,12], Etienne [7] and Vallet [18] for the mathematical aspect of the monolithological case and Eymard et al. [8] for a theoretical and numerical approach of an inverse problem.

In Section 2, we consider a variational inequality, involving a unilateral constraint on the outflow boundary.

After giving a brief presentation of the model and of the mathematical framework, we would be interested in the existence of a solution. The main result proves the existence of an approximating sequence via an implicit time-discretization scheme. Then some a priori estimates, independent of the time-discretized parameter, are presented. Passing to the limits in the approximate variational inequality is still an open problem, mainly because of a lack of compactness results.

\footnotetext{
Keywords and phrases. Stratigraphic models, weather limited, degenerated parabolic-hyperbolic conservation laws.

1 Departamento de Matemática, Universidade da Beira Interior Covilhã 6201-001, Portugal. anton@ubi.pt

2 Laboratoire de Mathématiques Appliquées UMR-CNRS 5142 IPRA BP 1155, 64013 Pau Cedex, France.

gerard.gagneux@univ-pau.fr, robert.luce@univ-pau.fr, guy.vallet@univ-pau.fr
}

(C) EDP Sciences, SMAI 2006 
Then, we present a concrete analysis of examples in the $1-D$ case, where, with some particular hypothesis, one is able to simplify the boundary conditions (and the problem) and overcome the difficulties following Gagneux et al. [10] idea. This section ends with numerical simulations and the presentation of open problems.

\section{An ORIGINAL HYPERBOLIC-PARABOLIC MODEL}

\subsection{Presentation of the model}

Let us consider a sedimentary basin whose base, denoted by $\Omega$, is a fixed domain of $\mathbb{R}^{d}(d=1,2$ in this framework) with a Lipschitzian boundary $\Gamma$. For any positive $T$, let us denote by $Q=] 0, T[\times \Omega$.

The sediment height, denoted by $u$, naturally satisfies the mass balance equation:

$$
\partial_{t} u+\operatorname{div}\{\vec{q}\}=0 \quad \text { in } Q,
$$

where $\vec{q}$, following a Darcy's law, is proportional to $\nabla u$, the gradient of the topography.

Moreover, a second phenomenon happens in a sedimentary basin formation process: sediments must first be produced in situ by weathering processes prior to be transported by surfacing erosion. Thus, Eymard et al. [14] introduce a maximum erosion rate $E$ such that:

$$
-\partial_{t} u \leq E \quad \text { in } Q
$$

where $E$ takes into account the composition, the structure and the age of the sediments.

The coupling of these two constraints is clearly an essential issue since both diffusive sedimentation or erosion and weather limited erosion can occur at the same time in a basin.

Then the authors introduce a new unknown $\lambda$ satisfying $0 \leq \lambda \leq 1$ and playing the role of a flux limiter, according to the Darcy's law:

$$
\vec{q}=-\lambda \nabla u \quad \text { in } Q .
$$

In order to give a mathematical modelling of $\lambda$, Gallouët proposes in [14] the following formulation:

$$
1-\lambda \geq 0, \partial_{t} u+E \geq 0,(1-\lambda)\left(\partial_{t} u+E\right)=0 \quad \text { a.e. in } Q .
$$

In other words, if one has to correct the flux, i.e. $\lambda<1$, then the constraint has to be observed, i.e. $\partial_{t} u+E=0$, otherwise $\lambda=1$.

Remark 2.1. If one assumes that $\lambda=1$ a.e. in $Q$, the mass balance law and the Darcy's law give $\partial_{t} u=\Delta u$ and one gets that $E+\Delta u \geq 0$. Then, if one considers a smooth function $E$, taking the limit when $t$ goes towards $0^{+}$in the sense of distributions leads to the fact that $\Delta u_{0}+E(0,$.$) is necessarily a non-negative Radon$ measure. Since this condition is not reasonable, $\lambda$ has to take values less than 1 . In that way, the modified flux approach is really required.

Boundary conditions. One considers an initial height given by: $u(0, x)=u_{0}(x), x \in \Omega$.

One assumes that $\Gamma$ is separated in two non-trivial distinct parts $\Gamma_{s}$ and $\Gamma_{e}$ in order to specify the output and the input fluxes.

The input flux is described by a matter transfer through $\Gamma_{e}$, proportional to the gradient of the topography:

$$
\lambda \nabla u . \vec{n}+f=0 \quad \text { on } \Gamma_{e}, \quad \text { with } f_{\mid \Gamma_{e}} \leq 0 .
$$

On the boundary $\Gamma_{s}$, one imposes the unilateral conditions (the out-flux has to be limited to satisfy the maximum erosion constraint):

$$
\lambda \nabla u \cdot \vec{n}+f \geq 0 ; \partial_{t} u+E \geq 0 ;(\lambda \nabla u \cdot \vec{n}+f)\left(\partial_{t} u+E\right)=0, t>0, \text { where } f_{\mid \Gamma_{s}} \geq 0 .
$$


Then, one has to look for a couple $(\lambda, u)$ that satisfies:

$$
\begin{gathered}
\partial_{t} u-\operatorname{div}\{\lambda \nabla u\}=0 \quad \text { a.e. in } Q, \\
u(0, .)=u_{0}, \quad \text { a.e. in } \Omega, \\
\lambda \nabla u \cdot \vec{n}+f=0 \quad \text { a.e. on }] 0, T\left[\times \Gamma_{e},\right. \\
\left.\lambda \nabla u \cdot \vec{n}+f \geq 0, \partial_{t} u+E \geq 0 \text { and }(\lambda \nabla u \cdot \vec{n}+f)\left(\partial_{t} u+E\right)=0, \text { a.e. on }\right] 0, T\left[\times \Gamma_{s},\right.
\end{gathered}
$$

and the global unilateral constraint:

$$
1-\lambda \geq 0, \partial_{t} u+E \geq 0,(1-\lambda)\left(\partial_{t} u+E\right)=0 \text { a.e. in } Q,
$$

where one assumes:

$$
\begin{aligned}
& u_{0} \in H^{1}(\Omega), \quad E \in L^{\infty}\left(0, T ; H^{1}(\Omega)\right) \text { satisfying } E \geq 0, \\
& f \in L^{2}(] 0, T[\times \Gamma) \text { where: }\left\{\begin{array}{l}
f \geq 0 \text { on }] 0, T\left[\times \Gamma_{s},\right. \\
f \leq 0 \text { on }] 0, T\left[\times \Gamma_{e} .\right.
\end{array}\right.
\end{aligned}
$$

Mathematical studies of such models have already been done through several papers. Let us cite for example:

(i) Antontsev et al. [3,4] and Gagneux et al. [12] where $\lambda$ is assumed to be a fixed parameter. In this paper, the authors prove that problem (1) to (4) is well-posed and propose a condition on $\lambda$ to obtain (5).

(ii) In the same papers, the authors present explicit solutions $(\lambda, u)$ to problem $(1)$ to $(5)$ in the $1-D$ dimensional space, by the way of travelling-waves techniques.

(iii) Eymard et al. [9] where $u$ is assumed to be given. The authors give an analytical and numerical analysis of the hyperbolic problem (1) to (5) of unknown $\lambda$.

(iv) Antontsev et al. [4], Gagneux et al. [10] and Vallet [18] concerning the problem (1), (2) and (5) with homogeneous Dirichlet boundary conditions. An original conservation law is proposed with partial results of existence. Then, in the $1-D$ sedimentary case (i.e. $E=0)$, explicit solutions and numerical simulations are proposed. The original mathematical formulation is: if $H$ denotes the maximal monotone graph of the Heaviside function (i.e. $H(x)=0$ if $x<0, H(x)=1$ if $x>0$ and $H(0)=[0,1])$, one looks for $(\lambda, u)$ such that:

$$
\partial_{t} u-\operatorname{div}\{\lambda \nabla u\}=0 \quad \text { a.e. } \operatorname{in} Q \quad \text { with } \quad \lambda \in H\left(\partial_{t} u+E\right)
$$

under the same boundary-initial conditions. The unilateral global constraint is then implicitly contained in the formulation (see Vallet [18] and Gagneux et al. [10] for information about this).

In the sequel, our goal is to extend the previous results to realistic boundary conditions and non-negative $E$.

\subsection{Definition of a solution}

Let us introduce the non-empty closed convex cone $\mathbb{K}=\left\{v \in H^{1}(\Omega) / v \geq 0\right.$ a.e. on $\left.\Gamma_{s}\right\}$.

At this moment, the variational formulation of the conservation law we are interested in, is:

Definition 2.2. A solution to the problem (1) to (5) is any couple $(\lambda, u)$ such that

$$
\left.u \in H^{1}(Q) \text { with } \partial_{t} u+E \in \mathbb{K} \text { a.e. } t \text { in }\right] 0, T\left[, \partial_{t} u+E \geq 0 \text { a.e.in } Q \quad \text { and } \quad \lambda \in L^{\infty}(Q) \cap H\left(\partial_{t} u+E\right)\right. \text {, }
$$

satisfying $u(0,)=.u_{0}$ a.e. in $\Omega$ and, for any $v \in \mathbb{K}$ and a.e. $t$ in $] 0, T[$,

$$
0 \leq \int_{\Omega} \partial_{t} u\left(v-\partial_{t} u-E\right) \mathrm{d} x+\int_{\Omega} \lambda \nabla u \cdot \nabla\left(v-\partial_{t} u-E\right) \mathrm{d} x+\int_{\Gamma} f\left(v-\partial_{t} u-E\right) \mathrm{d} \sigma .
$$


In other words, the resulting model appears to be a non-standard free boundary problem of the form:

$$
0 \in \partial_{t} u-\operatorname{div}\left\{H\left(\partial_{t} u+E\right) \nabla u\right\} .
$$

\section{Remark 2.3.}

(i) In the one space dimensional case $(d=1)$, the fact that the condition $\partial_{t} u+E \geq 0$ a.e. in $Q$ is implicit to the formulation can be proved, else a conjecture has to be stated (see Gagneux et al. [10]).

(ii) As mentioned in Vallet [18] and in Proposition 2.20, the solution to such a problem is not a priori unique. Therefore, one is interested in the maximal solution $(\lambda, u)$ with respect to the multiplier $\lambda$, i.e. if $(\mu, v)$ is any other solution, then $\mu \leq \lambda$. On the other hand, the existence of such a solution is an open problem.

\subsection{Mathematical study of the conservation law}

This section is devoted to the analysis of an implicit time-discretization scheme. An existence result of such a sequence of approximation and some a priori estimates are presented. The section ends with the presentation of the difficulties in passing to the limits in the variational inequality.

Let us start with a remark concerning the regularity of the solution we are looking for.

Remark 2.4. Let us consider the general problem: find $u \in H^{1}(Q)$ such that $u(0,)=.u_{0}$, a.e. in $\Omega$, and, for any $v \in H^{1}(\Omega)$,

$$
\left.\int_{\Omega}\left\{\partial_{t} u v+a\left(\partial_{t} u\right) \nabla u . \nabla v\right\} \mathrm{d} x=0, \quad t \text { a.e. in }\right] 0, T[,
$$

where $a$ is a lipschitzian-continuous function with $0 \leq a() \leq M<.\infty$.

Let us assume informally that $v_{\varepsilon}=\int_{0}^{\partial_{t} u} \frac{\mathrm{d} s}{a(s)+\varepsilon}(\varepsilon>0)$ is an admissible test-function in the previous equation. Then, one obtains: $\frac{1}{M}\left(\left\|\partial_{t} u\right\|_{L^{2}(Q)}^{2}+\left\|\operatorname{div}\left(a\left(\partial_{t} u\right) \nabla u\right)\right\|_{L^{2}(Q)}^{2}\right)+\|u\|_{L^{\infty}\left(0, T ; H^{1}(\Omega)\right)}^{2} \leq\left\|u_{0}\right\|_{H^{1}(\Omega)}^{2}$.

Note that in a time-discretization method, the discrete version of $v_{\epsilon}$ is an admissible test-function.

Usually, the existence of a solution to such problem is proved by the way of an implicit time-discretization scheme. The heart of the following proofs is to state: for any positive integer $N$, if $h=\frac{T}{N}$, there exists a sequence $\left\{\left(\lambda_{k}, u^{k}\right)\right\}_{k \geq 0}$ such that $u^{0}=u_{0}$,

$$
u^{k} \in H^{1}(\Omega), \quad \frac{u^{k}-u^{k-1}}{h}+E_{k} \in \mathbb{K}, \quad \lambda_{k} \in L^{\infty}(\Omega), \quad \lambda_{k} \in H\left(\frac{u^{k}-u^{k-1}}{h}+E_{k}\right),
$$

and, by denoting $E_{k}(x)=\frac{1}{h} \int_{(k-1) h}^{k h} E(t, x) \mathrm{d} t$ and $f_{k}(x)=\frac{1}{h} \int_{(k-1) h}^{k h} f(t, x) \mathrm{d} t$, for any $v \in \mathbb{K}$, one gets

$$
\begin{aligned}
0 & \leq \int_{\Omega} \frac{u^{k}-u^{k-1}}{h}\left(v-\frac{u^{k}-u^{k-1}}{h}-E_{k}\right) \mathrm{d} x \\
& +\int_{\Omega} \lambda_{k} \nabla u^{k} \cdot \nabla\left(v-\frac{u^{k}-u^{k-1}}{h}-E_{k}\right) \mathrm{d} x+\int_{\Gamma} f_{k} \cdot\left(v-\frac{u^{k}-u^{k-1}}{h}-E_{k}\right) \mathrm{d} \sigma .
\end{aligned}
$$

In order to prove the existence of such a sequence, a technique of artificial viscosity and a method of penalization of the constraint on the boundary $\Gamma_{s}$ is proposed. Note that, one only needs to establish the result for the first iteration. The sequence will be obtained by induction.

Let us consider the following assumptions on the data for the first iteration:

$$
\begin{aligned}
(H):\left\{\begin{array}{l}
E_{1} \in H^{1}(\Omega), E_{1} \geq 0, u_{0} \in H^{1}(\Omega), \\
\left.f_{1} \in L^{2}(\Gamma) \text { such that } f_{1} \geq 0 \text { on }\right] 0, T\left[\times \Gamma_{s} \quad \text { and } \quad f_{1} \leq 0 \quad \text { on }\right] 0, T\left[\times \Gamma_{e} .\right.
\end{array}\right. \\
\forall \varepsilon \in] 0,1\left[, a_{\varepsilon}(x)=\max \left(\epsilon, \min \left(1, \frac{1-\varepsilon}{\varepsilon} x+1\right)\right) .\right.
\end{aligned}
$$


Lemma 2.5. Considering $(H)$ and any $\varepsilon, \delta, h$ in $] 0,1\left[\right.$, there exists a unique element $u_{\varepsilon \delta}$ in $H^{1}(\Omega)$ such that,

$\forall v \in H^{1}(\Omega), 0=\int_{\Omega}\left\{\frac{u_{\varepsilon \delta}-u_{0}}{h} v+a_{\varepsilon}\left(\frac{u_{\varepsilon \delta}-u_{0}}{h}+E_{1}\right) \nabla u_{\varepsilon \delta} . \nabla v\right\} \mathrm{d} x+\int_{\Gamma} f_{1} v \mathrm{~d} \sigma-\frac{1}{\delta} \int_{\Gamma_{s}}\left(\frac{u_{\varepsilon \delta}-u_{0}}{h}+E_{1}\right)^{-} v \mathrm{~d} \sigma$.

Proof. For any $S$ in $H^{1}(\Omega)$, the theory of monotone operators (cf. Lions [17]) leads to the existence and the uniqueness of the solution to the following problem: find $u_{S, \varepsilon, \delta}$ in $H^{1}(\Omega)$ such that, for any $v \in H^{1}(\Omega)$,

$0=\int_{\Omega}\left\{\frac{u_{S, \varepsilon, \delta}-u_{0}}{h} v+a_{\varepsilon}\left(\frac{S-u_{0}}{h}+E_{1}\right) \nabla u_{S, \varepsilon, \delta} . \nabla v\right\} \mathrm{d} x+\int_{\Gamma} f_{1} v \mathrm{~d} \sigma-\frac{1}{\delta} \int_{\Gamma_{s}}\left(\frac{u_{S, \varepsilon, \delta}-u_{0}}{h}+E_{1}\right)^{-} v \mathrm{~d} \sigma$.

Considering the application $\Psi: H^{1}(\Omega) \rightarrow H^{1}(\Omega), S \mapsto u_{S, \varepsilon, \delta}$, the fixed-point theorem of Schauder-Tikhonov in the separable hilbertian framework (see Gagneux et al. [11] pp. 29-30) leads to the existence of a solution. The uniqueness is based on a $L^{1}$ - method by using a suitable approximation of the sign function.

Lemma 2.6. Considering $(H)$ and any $\varepsilon, h$ in $] 0,1\left[\right.$, there exists a unique element $u_{\varepsilon}$ in $H^{1}(\Omega)$ such that: $u_{\varepsilon} \geq u_{0}-h E_{1}$ a.e. on $\Gamma_{s}$ and, for any $v \in \mathbb{K}$ :

$$
\begin{aligned}
0 \leq & \int_{\Omega} \frac{u_{\varepsilon}-u_{0}}{h}\left(v-\frac{u_{\varepsilon}-u_{0}}{h}-E_{1}\right) v \mathrm{~d} x \\
& +\int_{\Omega} a_{\varepsilon}\left(\frac{u_{\varepsilon}-u_{0}}{h}+E_{1}\right) \nabla u_{\varepsilon} \cdot \nabla\left(v-\frac{u_{\varepsilon}-u_{0}}{h}-E_{1}\right) \mathrm{d} x+\int_{\Gamma} f_{1}\left(v-\frac{u_{\varepsilon}-u_{0}}{h}-E_{1}\right) \mathrm{d} \sigma .
\end{aligned}
$$

Proof. Passing to the limits with $\delta$ is classical (see Lions [17] p. 372 concerning penalization problems) and provides the existence of an element $u_{\varepsilon}$.

For the uniqueness of the solution, if one denotes by $w_{\varepsilon}=\frac{u_{\varepsilon}-u_{0}}{h}+E_{1}, w_{\varepsilon}$ is a solution to the problem

$$
0 \leq \int_{\Omega} w_{\varepsilon}\left(v-w_{\varepsilon}\right) v \mathrm{~d} x+\int_{\Omega} a_{\varepsilon}\left(w_{\varepsilon}\right) \nabla u_{\varepsilon} \cdot \nabla\left(v-w_{\varepsilon}\right) \mathrm{d} x+\int_{\Gamma} f_{1}\left(v-w_{\varepsilon}\right) \mathrm{d} \sigma+\int_{\Omega} a_{\varepsilon}\left(w_{\varepsilon}\right) \nabla\left(u_{0}-h E_{1}\right) . \nabla v \mathrm{~d} x .
$$

Since $w_{\varepsilon}$ is unique by using a $L^{1}$ - method for variational inequalities thanks to a suitable approximation of the sign function, $u_{\varepsilon}$ is unique too. The reader interested by the technical details of the demonstrations would find some information in Antontsev et al. [1].

In order to pass to the limits with respect to $\varepsilon$, the following a priori estimates are needed.

Lemma 2.7. Considering $(H)$ and any $\varepsilon, h$ in $] 0,1[$, there exists a positive constant $C$ such that (i) $\left\|w_{\varepsilon}\right\|_{L^{2}(\Omega)} \leq C, \quad$ (ii) $\int_{\Omega} a_{\varepsilon}\left(w_{\varepsilon}\right)\left|\nabla w_{\varepsilon}\right|^{2} \mathrm{~d} x \leq C$, and $\quad$ (iii) $\left\|w_{\varepsilon}^{+}\right\|_{H^{1}(\Omega)} \leq C$, where $w_{\varepsilon}=\frac{u_{\varepsilon}-u_{0}}{h}+E_{1}$.

Proof. Since $\mathbb{K}$ is a convex cone containing 0, (7) leads to

$$
\begin{array}{ll} 
& \int_{\Omega}\left\{w_{\varepsilon}^{2}+h a_{\varepsilon}\left(w_{\varepsilon}\right)\left|\nabla w_{\varepsilon}\right|^{2}\right\} \mathrm{d} x \leq-\left(I_{1}+I_{2}+I_{3}\right), \\
\text { where } & I_{1}=\int_{\Omega} E_{1} w_{\varepsilon} \mathrm{d} x, \quad I_{2}=\int_{\Omega} a_{\varepsilon}\left(w_{\varepsilon}\right) \nabla\left(u_{0}-h E_{1}\right) . \nabla w_{\varepsilon} \mathrm{d} x \quad \text { and } \quad I_{3}=\int_{\Gamma} f_{1} w_{\varepsilon} \mathrm{d} \sigma .
\end{array}
$$

Moreover, since $f_{1} \geq 0, w_{\varepsilon} \geq 0$ on $\Gamma_{s}$ and $f_{1} \leq 0$ on $\Gamma_{e}$, embedding theorems and Cauchy's inequality lead to:

$$
\begin{aligned}
-I_{3} & =-\left|\int_{\Gamma_{s}} f_{1} w_{\varepsilon} \mathrm{d} \sigma\right|-\left|\int_{\Gamma_{e}} f_{1} w_{\varepsilon}^{-} \mathrm{d} \sigma\right|+\int_{\Gamma_{e}}\left|f_{1}\right| w_{\varepsilon}^{+} \mathrm{d} \sigma \leq \frac{\mu h}{2} \int_{\Omega}\left(w_{\varepsilon}^{+2}+\left|\nabla w_{\varepsilon}^{+}\right|^{2}\right) \mathrm{d} x+\frac{1}{2 \mu h} \int_{\Gamma_{e}} f_{1}^{2} \mathrm{dx} \\
& \leq \frac{\mu}{2} \int_{\Omega}\left(w_{\varepsilon}^{2}+h a_{\varepsilon}\left(w_{\varepsilon}\right)\left|\nabla w_{\varepsilon}\right|^{2}\right) \mathrm{d} x+\frac{1}{2 \mu} \int_{\Gamma_{e}} f_{1}^{2} \mathrm{~d} x,
\end{aligned}
$$


since $\int_{\Omega}\left|\nabla w_{\varepsilon}^{+}\right|^{2} \mathrm{~d} x \leq \int_{\Omega} a_{\varepsilon}\left(w_{\varepsilon}\right)\left|\nabla w_{\varepsilon}\right|^{2} \mathrm{~d} x$ according to (6). Next, using Cauchy's inequality, one gets that:

$$
\begin{aligned}
& \left|I_{1}\right| \leq \frac{\mu}{2} \int_{\Omega} w_{\varepsilon}^{2} \mathrm{~d} x+\frac{1}{2 \mu} \int_{\Omega} E_{1}^{2} \mathrm{~d} x \\
& \left|I_{2}\right| \leq \mu \int_{\Omega} h a_{\varepsilon}\left(w_{\varepsilon}\right)\left|\nabla w_{\varepsilon}\right|^{2} \mathrm{~d} x+\frac{1}{2 \mu} \int_{\Omega} h a_{\varepsilon}\left(w_{\varepsilon}\right) E_{1}^{2} \mathrm{~d} x+\frac{1}{2 \mu} \int_{\Omega} a_{\varepsilon}\left(w_{\varepsilon}\right) \frac{\left|\nabla u_{0}\right|^{2}}{h} \mathrm{~d} x .
\end{aligned}
$$

Joining last estimates with suitable choice of $\mu$ in $] 0,1[$, we come to

$$
\int_{\Omega}\left\{w_{\varepsilon}^{2}+h a_{\varepsilon}\left(w_{\varepsilon}\right)\left|\nabla w_{\varepsilon}\right|^{2}\right\} \mathrm{d} x \leq C(\mu)\left(\int_{\Omega}\left(\left(h a_{\varepsilon}\left(w_{\varepsilon}\right)+1\right) E_{1}^{2}+a_{\varepsilon}\left(w_{\varepsilon}\right) \frac{\left|\nabla u_{0}\right|^{2}}{h}\right) \mathrm{d} x+\int_{\Gamma_{e}} f_{1}^{2} \mathrm{~d} x\right) .
$$

Under assumption $0<h<1$, the last estimate can be rewritten in the form

$$
\int_{\Omega}\left\{w_{\varepsilon}^{2}+h a_{\varepsilon}\left(w_{\varepsilon}\right)\left|\nabla w_{\varepsilon}\right|^{2}\right\} \mathrm{d} x \leq C\left(\frac{1}{h}\right)\left(\left\|E_{1}\right\|_{L^{2}(\Omega)}^{2}+\left\|u_{0}\right\|_{H^{1}(\Omega)}^{2}+\left\|f_{1}\right\|_{L^{2}\left(\Gamma_{e}\right)}^{2}\right) .
$$

Then, one gets points i) and ii), and $\left(w_{\varepsilon}^{+}\right)_{\varepsilon}$ is bounded in $H^{1}(\Omega)$, since $a_{\varepsilon}=1$ in $\mathbb{R}^{+}$.

Lemma 2.8. Considering $(H)$ and any $\varepsilon, h$ in $] 0,1\left[\right.$, there exists a subsequence, still denoted by $\left(w_{\varepsilon}\right)_{\varepsilon}$, such that, when $\epsilon$ goes to $0^{+}$,

(i) $\left(w_{\varepsilon}+\varepsilon\right)^{-}:=\max \left(0,-w_{\varepsilon}-\varepsilon\right)$ weakly converges towards 0 in $H^{1}(\Omega) ; \quad$ (ii) $w_{\varepsilon}^{-}$converges towards 0 in $L^{2}(\Omega)$; (iii) $w_{\varepsilon}^{-}$converges towards 0 in $L^{2}(\Gamma)$.

Proof. Let us set $v=w_{\varepsilon}+\left(w_{\varepsilon}+\varepsilon\right)^{-}$in (7). Then, one has:

$$
\begin{aligned}
0 \geq & -\int_{\Omega}\left\{\left(w_{\varepsilon}+\varepsilon\right)\left(-w_{\varepsilon}-\varepsilon\right)^{+}-(E+\varepsilon)\left(-w_{\varepsilon}-\varepsilon\right)^{+}\right\} \mathrm{d} x-\int_{\Gamma} f_{1}\left(-w_{\varepsilon}-\varepsilon\right)^{+} \mathrm{d} \sigma \\
& -\int_{\Omega} h a_{\varepsilon}\left(w_{\varepsilon}\right) \nabla w_{\varepsilon} \cdot \nabla\left(-w_{\varepsilon}-\varepsilon\right)^{+} \mathrm{d} x-\int_{\Omega} a_{\varepsilon}\left(w_{\varepsilon}\right) \nabla\left(u_{0}-h E_{1}\right) \cdot \nabla\left(-w_{\varepsilon}-\varepsilon\right)^{+} \mathrm{d} x .
\end{aligned}
$$

Remark that $-\int_{\Omega \cap\left\{w_{\varepsilon} \leq-\varepsilon\right\}} a_{\varepsilon}\left(w_{\varepsilon}\right) \nabla w_{\varepsilon} \cdot \nabla\left(-w_{\varepsilon}-\varepsilon\right)^{+} \mathrm{d} x=\varepsilon \int_{\Omega}\left|\nabla\left(w_{\varepsilon}+\varepsilon\right)^{-}\right|^{2} \mathrm{~d} x$.

Since $E+\varepsilon \geq 0$, a constant $C=C\left(\left\|\nabla\left(u_{0}-h E_{1}\right)\right\|_{L^{2}(\Omega)}\right)$ exists such that

$$
\int_{\Omega}\left|\left(w_{\varepsilon}+\varepsilon\right)^{-}\right|^{2} \mathrm{~d} x+\frac{\varepsilon h}{2} \int_{\Omega}\left|\nabla\left(w_{\varepsilon}+\varepsilon\right)^{-}\right|^{2} \mathrm{~d} x-\int_{\Gamma} f_{1}\left(-w_{\varepsilon}-\varepsilon\right)^{+} \mathrm{d} \sigma \leq C \varepsilon .
$$

Since $w_{\varepsilon} \geq 0$ a.e. in $\Gamma_{s}$ and $f_{1} \leq 0$ on $\Gamma_{e}$, it follows that

$$
-\int_{\Gamma} f_{1}\left(-w_{\varepsilon}-\varepsilon\right)^{+} \mathrm{d} \sigma=-\int_{\Gamma_{e}} f_{1}\left(-w_{\varepsilon}-\varepsilon\right)^{+} \mathrm{d} \sigma \geq 0
$$

So, one gets that

$$
\int_{\Omega}\left|\left(w_{\varepsilon}+\varepsilon\right)^{-}\right|^{2} \mathrm{~d} x+\varepsilon h \int_{\Omega}\left|\nabla\left(w_{\varepsilon}+\varepsilon\right)^{-}\right|^{2} \mathrm{~d} x \leq C \varepsilon .
$$

Thus, $\left(w_{\varepsilon}+\varepsilon\right)^{-}$is bounded in $H^{1}(\Omega)$, and when $\varepsilon$ goes to $0^{+},\left(w_{\varepsilon}+\varepsilon\right)^{-}$converges towards 0 in $L^{2}(\Omega)$. So $\left(w_{\varepsilon}\right)^{-}$converges towards 0 in $L^{2}(\Omega)$ and $\left(w_{\varepsilon}+\varepsilon\right)^{-}$converges weakly towards 0 in $H^{1}(\Omega)$. Therefore, $\left(w_{\varepsilon}+\varepsilon\right)^{-}$ converges strongly towards 0 in $H^{s}(\Omega)$ for any given $\frac{1}{2}<s<1$, and $\left(w_{\varepsilon}+\varepsilon\right)^{-}$converges towards 0 in $L^{2}(\Gamma)$ as well as $\left(w_{\varepsilon}\right)^{-}$. 
Lemma 2.9. Considering $(H)$ and $h$ in $] 0,1\left[\right.$, there exists $u$ in $H^{1}(\Omega)$ and a subsequence, still denoted by $\left(w_{\varepsilon}\right)_{\varepsilon}$, such that when $\varepsilon$ goes towards $0^{+}$,

$$
\begin{aligned}
& A_{\varepsilon}\left(\frac{u_{\varepsilon}-u_{0}}{h}+E_{1}\right) \quad \text { converges weakly towards }\left(\frac{u-u_{0}}{h}+E_{1}\right)^{+} \text {in } H^{1}(\Omega) \text { and } \\
& G_{\varepsilon}\left(\frac{u_{\varepsilon}-u_{0}}{h}+E_{1}\right) \quad \text { converges weakly towards }\left(\frac{u-u_{0}}{h}+E_{1}\right)^{+} \text {in } H^{1}(\Omega),
\end{aligned}
$$

where $A_{\varepsilon}(x)=\int_{0}^{x} a_{\varepsilon}(s) \mathrm{d} s$ and $G_{\varepsilon}(x)=\int_{0}^{x} \sqrt{a_{\varepsilon}(s)} \mathrm{d} s$.

Proof. Thanks to lemma 2.7, up to a subsequence still indexed by $\varepsilon, w_{\varepsilon}^{+}$converges, weakly in $H^{1}(\Omega)$ and strongly in $L^{2}(\Omega)$ towards $w$ with $w \geq 0$ a.e. in $\Omega$. Moreover, $w_{\varepsilon}^{-}$converges strongly towards 0 in $L^{2}(\Omega)$, so $w_{\varepsilon}$ converges strongly towards $w$ in $L^{2}(\Omega)$. Let us denote $u=u_{0}+h\left(w-E_{1}\right)$. Then, $u \in H^{1}(\Omega)$ and the lemma is obtained by computation and thanks to the previous a priori estimates.

Lemma 2.10. Considering $(H), h$ in $] 0,1\left[\right.$ and $u$ given by the previous lemma, there exists $\lambda_{1}$ in $L^{\infty}(\Omega)$ and a subsequence still denoted by $\left(u_{\varepsilon}\right)_{\varepsilon}$, such that $a_{\varepsilon}\left(\frac{u_{\varepsilon}-u_{0}}{h}+E_{1}\right)$ converges weakly towards $\lambda_{1}$ in $L^{\infty}(Q)$ weak-* when $\varepsilon$ goes to $0^{+}$, with $\lambda_{1} \in H\left(\frac{u-u_{0}}{h}+E_{1}\right)$.

Proof. Since $\frac{u_{\varepsilon}-u_{0}}{h}+E_{1}$ converges towards $\frac{u-u_{0}}{h}+E_{1}$ in $L^{2}(\Omega)$ with $\varepsilon$ towards $0^{+}$, it converges a.e. in $\Omega$ for a subsequence. Moreover $0 \leq a_{\varepsilon} \leq 1$, so $a_{\varepsilon}\left(\frac{u_{\varepsilon}-u_{0}}{h}+E_{1}\right)$ converges weakly towards $\lambda_{1}$ in $L^{\infty}(\Omega)$ weak-* with $0 \leq \lambda_{1} \leq 1$ a.e. in $\Omega$.

Then, by construction, denoting by

$$
\begin{aligned}
& A=\left\{x \in \Omega /\left(\frac{u_{\varepsilon}-u_{0}}{h}+E_{1}\right)(x) \rightarrow\left(\frac{u-u_{0}}{h}+E_{1}\right)(x) \text { and }\left(\frac{u-u_{0}}{h}+E_{1}\right)(x) \neq 0\right\}, \\
& \text { and } \quad A^{+}=\left\{x \in A / \frac{u-u_{0}}{h}+E_{1}>0\right\}, \quad A^{-}=\left\{x \in A / \frac{u-u_{0}}{h}+E_{1}<0\right\},
\end{aligned}
$$

$a_{\varepsilon}\left(\frac{u_{\varepsilon}-u_{0}}{h}+E_{1}\right)$ converges a.e. in $A$ towards $\operatorname{sign}_{0}^{+}\left(\frac{u-u_{0}}{h}+E_{1}\right)$ and $\lambda_{1} \in H\left(\frac{u-u_{0}}{h}+E_{1}\right)$.

Lemma 2.11. Considering $(H), h$ in $] 0,1\left[, u\right.$ and $\lambda_{1}$ given by the previous lemma and a subsequence still denoted by $\left(u_{\varepsilon}\right)_{\varepsilon}$, one has:

$$
\limsup _{\varepsilon \rightarrow 0^{+}} \int_{\Omega} a_{\varepsilon}\left(\frac{u_{\varepsilon}-u_{0}}{h}+E_{1}\right) \nabla u_{\varepsilon} \cdot \nabla\left(v-\frac{u_{\varepsilon}-u_{0}}{h}-E_{1}\right) \mathrm{d} x \leq \int_{\Omega} \lambda_{1} \nabla u \cdot \nabla\left(v-\frac{u-u_{0}}{h}-E_{1}\right) \mathrm{d} x .
$$

Proof. One writes that:

$$
\begin{aligned}
I_{\varepsilon} & =\int_{\Omega} a_{\varepsilon}\left(\frac{u_{\varepsilon}-u_{0}}{h}+E_{1}\right) \nabla u_{\varepsilon} \cdot \nabla\left(v-\frac{u_{\varepsilon}-u_{0}}{h}-E_{1}\right) \mathrm{d} x \\
& =h \int_{\Omega} a_{\varepsilon}\left(w_{\varepsilon}\right) \nabla w_{\varepsilon} \cdot \nabla\left(v-w_{\varepsilon}\right) \mathrm{d} x+\int_{\Omega} a_{\varepsilon}\left(w_{\varepsilon}\right) \nabla\left(u_{0}-h E_{1}\right) \cdot \nabla\left(v-w_{\varepsilon}\right) \mathrm{d} x \\
& =h \int_{\Omega} \nabla A_{\varepsilon}\left(w_{\varepsilon}\right) \cdot \nabla v \mathrm{~d} x-h \int_{\Omega}\left|\nabla G_{\varepsilon}\left(w_{\varepsilon}\right)\right|^{2} \mathrm{~d} x+\int_{\Omega} a_{\varepsilon}\left(w_{\varepsilon}\right) \nabla\left(u_{0}-h E_{1}\right) \cdot \nabla\left(v-w_{\varepsilon}\right) \mathrm{d} x .
\end{aligned}
$$

Thus, passing to the limits gives:

$$
\begin{aligned}
\limsup _{\varepsilon \rightarrow 0^{+}} I_{\varepsilon} \leq & h \int_{\Omega} \nabla\left(\frac{u-u_{0}}{h}+E_{1}\right)^{+} \cdot \nabla v \mathrm{~d} x-h \int_{\Omega}\left|\nabla\left(\frac{u-u_{0}}{h}+E_{1}\right)^{+}\right|^{2} \mathrm{~d} x \\
& +\int_{\Omega} \nabla\left(u_{0}-h E_{1}\right) \cdot \nabla\left(\frac{u-u_{0}}{h}+E_{1}\right)^{+} \mathrm{d} x+\int_{\Omega} \lambda_{1} \nabla\left(u_{0}-h E_{1}\right) \cdot \nabla v \mathrm{~d} x .
\end{aligned}
$$


and the result holds since, thanks to the lemma of Saks, $\nabla\left(\frac{u-u_{0}}{h}+E_{1}\right)^{+}=\lambda_{1} \nabla\left(\frac{u-u_{0}}{h}+E_{1}\right)$ a.e.

All these lemmata lead us to the following proposition:

Proposition 2.12. Considering $(H)$ and $h$ in $] 0,1\left[\right.$, there exists a couple $\left(\lambda_{1}, u^{1}\right)$ such that:

$$
u^{1} \in H^{1}(\Omega), \frac{u^{1}-u_{0}}{h}+E_{1} \geq 0 \quad \text { a.e. in } \Omega \text { and on } \Gamma_{s}, \quad \lambda_{1} \in L^{\infty}(\Omega), \lambda_{1} \in H\left(\frac{u^{1}-u_{0}}{h}+E_{1}\right)
$$

which satisfies, for any $v \in \mathbb{K}$ :

$0 \leq \int_{\Omega} \frac{u^{1}-u_{0}}{h}\left(v-\frac{u^{1}-u_{0}}{h}-E_{1}\right) \mathrm{d} x+\int_{\Omega} \lambda_{1} \nabla u^{1} \cdot \nabla\left(v-\frac{u^{1}-u_{0}}{h}-E_{1}\right) \mathrm{d} x+\int_{\Gamma} f_{1} \cdot\left(v-\frac{u^{1}-u_{0}}{h}-E_{1}\right) \mathrm{d} \sigma$.

By induction of this proposition, one is then able to conclude:

Theorem 2.13. Let us consider a positive integer $N$ such that $h=\frac{T}{N}<1, \forall k=1, . ., N, E_{k} \in H^{1}(\Omega)$ with $E_{k} \geq 0, f_{k} \in L^{2}(\Gamma)$ with $f_{k} \leq 0$ on $\Gamma_{e}$ and $f_{k} \geq 0$ on $\Gamma_{s}$ and $u_{0} \in H^{1}(\Omega)$. Then, there exists a sequence $\left\{\left(\lambda_{k}, u^{k}\right)\right\}_{k}$ such that:

$$
\begin{aligned}
& \lambda_{k} \in L^{\infty}(\Omega), \lambda_{k} \in H\left(\frac{u^{k}-u^{k-1}}{h}+E_{k}\right), \\
& u^{0}=u_{0}, u^{k} \in H^{1}(\Omega), \frac{u^{k}-u^{k-1}}{h}+E_{k} \geq 0 \text { a.e. in } \Omega \text { and on } \Gamma_{s}
\end{aligned}
$$

and, $\forall v \in \mathbb{K}, \quad 0 \leq \int_{\Omega} \frac{u^{k}-u^{k-1}}{h}\left(v-\frac{u^{k}-u^{k-1}}{h}-E_{k}\right) \mathrm{d} x$

$$
+\int_{\Omega} \lambda_{k} \nabla u^{k} \cdot \nabla\left(v-\frac{u^{k}-u^{k-1}}{h}-E_{k}\right) \mathrm{d} x+\int_{\Gamma} f_{k} \cdot\left(v-\frac{u^{k}-u^{k-1}}{h}-E_{k}\right) \mathrm{d} \sigma .
$$

Let us give now some a priori estimates that should allow us to pass to limits with $h$ towards $0^{+}$.

Lemma 2.14. Considering the previous theorem hypothesis with $f_{e}=0$ and $h<\frac{1}{2}$, for any integer $n$, one has:

$\frac{1}{h} \sum_{k=1}^{n}\left\|u^{k}-u^{k-1}\right\|_{L^{2}(\Omega)}^{2}+\left\|u^{n}\right\|_{H^{1}(\Omega)}^{2}+\sum_{k=1}^{n}\left\|u^{k}-u^{k-1}\right\|_{H^{1}(\Omega)}^{2} \leq\left(1+2 e^{2}\right)\left[\left\|\nabla u_{0}\right\|_{\left(L^{2}(\Omega)\right)^{d}}^{2}+h \sum_{k=1}^{N}\left\|E_{k}\right\|_{H^{1}(\Omega)}^{2}\right]$.

Proof. Let us denote $w_{k}=\frac{u^{k}-u^{k-1}}{h}+E_{k}$. Since $v=2 w_{k}$ and $v=0$ are available test-functions, one gets:

$$
0=\int_{\Omega}\left\{\left(w_{k}-E_{k}\right) w_{k}+\lambda^{k} \nabla u^{k} \cdot \nabla w_{k}\right\} \mathrm{d} x+\int_{\Gamma} f_{k} w_{k} \mathrm{~d} \sigma .
$$

Since $\lambda^{k} \in H\left(w_{k}\right)$ with $w_{k} \geq 0$, one has $\lambda^{k} \nabla u^{k} . \nabla w_{k}=\nabla u^{k} . \nabla w_{k}{ }^{+}=\nabla u^{k} . \nabla w_{k}$ and one gets

$$
\int_{\Omega}\left|w_{k}\right|^{2} \mathrm{~d} x-\int_{\Omega} E_{k} w_{k} \mathrm{~d} x+\frac{1}{2 h} \int_{\Omega}\left[\left|\nabla u^{k}\right|+\left|\nabla\left(u^{k}-u^{k-1}\right)\right|^{2}-\left|\nabla u^{k-1}\right|^{2}\right] \mathrm{d} x=-\int_{\Gamma} f_{k} w_{k} \mathrm{~d} \sigma-\int_{\Omega} \nabla u^{k} . \nabla E_{k} \mathrm{~d} x .
$$


Since $\int_{\Gamma} f_{k} w_{k} \mathrm{~d} \sigma=\int_{\Gamma_{s}} f_{k} w_{k} \mathrm{~d} \sigma \geq 0$, this leads, thanks to some Young's inequalities, to

$$
\begin{aligned}
\frac{1}{h} \sum_{k=1}^{n}\left\|u^{k}-u^{k-1}\right\|_{L^{2}(\Omega)}^{2}+\left\|\nabla u^{n}\right\|_{\left(L^{2}(\Omega)\right)^{d}}^{2}+\sum_{k=1}^{n}\left\|\nabla\left(u^{k}-u^{k-1}\right)\right\|_{\left(L^{2}(\Omega)\right)^{d}}^{2} & \leq\left\|\nabla u_{0}\right\|_{\left(L^{2}(\Omega)\right)^{d}}^{2}+h \sum_{k=1}^{n}\left\|\nabla u^{k}\right\|_{\left(L^{2}(\Omega)\right)^{d}}^{2}+h \sum_{k=1}^{n}\left\|E_{k}\right\|_{H^{1}(\Omega)}^{2} .
\end{aligned}
$$

Thanks to the discrete Gronwall lemma (see Lions [16]), one gets $\left(h<\frac{1}{2}\right)$ finally

$$
\left\|\nabla u^{n}\right\|_{\left(L^{2}(\Omega)\right)^{d}}^{2} \leq 2 e^{2}\left[\left\|\nabla u_{0}\right\|_{\left(L^{2}(\Omega)\right)^{d}}^{2}+h \sum_{k=1}^{N}\left\|E_{k}\right\|_{H^{1}(\Omega)}^{2}\right]
$$

The previous lemma leads us to the following proposition:

Proposition 2.15. Considering the hypothesis of the previous theorem with $f_{e}=0$ and $h<\frac{1}{2}$, if one denotes

$$
\begin{gathered}
E_{h}(x)=\sum_{k=0}^{k=N-1} E_{k+1} \cdot 1_{[k h,(k+1) h[}, \quad f_{h}(x)=\sum_{k=0}^{k=N-1} f_{k+1} \cdot 1_{[k h,(k+1) h[}, \\
\hat{u}_{h}(x)=\sum_{k=0}^{k=N-1}\left[\frac{u^{k+1}-u^{k}}{h}(t-k h)+u^{k}\right] .1_{[k h,(k+1) h[} \quad \text { and } \quad \lambda_{h}(x)=\sum_{k=0}^{k=N-1} \lambda^{k+1} \cdot 1_{[k h,(k+1) h[},
\end{gathered}
$$

then, one gets, for any $v$ in $L^{2}(0, T ; \mathbb{K})$,

$$
0 \leq \int_{\Omega} \partial_{t} \hat{u}_{h}\left(v-\partial_{t} \hat{u}_{h}-E_{h}\right) \mathrm{d} x+\int_{\Omega} \lambda_{h} \nabla u_{h} . \nabla\left(v-\partial_{t} \hat{u}_{h}-E_{h}\right) \mathrm{d} x+\int_{\Gamma} f_{h}\left(v-\partial_{t} \hat{u}_{h}-E_{h}\right) \mathrm{d} \sigma .
$$

The sequence $\left(\hat{u}_{h}\right)$ is bounded in $H^{1}(Q) \cap L^{\infty}\left(0, T ; H^{1}(\Omega)\right)$. Thus, it is relatively compact in $C\left([0, T] ; L^{2}(\Omega)\right)$ and a mild solution exists in the sense of Bénilan et al. [5].

Remark 2.16. Unfortunately, passing to the limits in the above inequality with respect to $h$ is still an open problem, mainly for two reasons:

(i) the presence of two weak convergences does not allow us to pass to the limits in the term $\int_{\Omega} \lambda_{h} \nabla \hat{u}_{h} . \nabla v \mathrm{~d} x$ when $h \rightarrow 0^{+}$, without some additional properties (see Gagneux et al. [10] for information about that),

(ii) one needs a priori estimates for $\partial_{t} \hat{u}_{h}$ in $L^{2}\left(0, T ; H^{1}(\Omega)\right)$ too, in order to consider the condition on $\Gamma_{s}$.

\subsection{Explicit solution in the $1-D$ case}

By considering particular assumptions, let us overcome in the $1-D$ case the problems of the previous remark. In this section, $\Omega=] 0,1\left[, \Gamma_{e}=\{0\}, \Gamma_{s}=\{1\}\right.$ and $f_{s},-f_{e}$ and $E$ are assumed to be non-negative constants. One is interested in the following problem: find $u \in H^{1}(0,1)$ and $\lambda \in H\left(\frac{u-u_{0}}{h}+E\right) \cap L^{\infty}(0,1)$ such that

$$
\begin{aligned}
\left.u-u_{0}-h\left(\lambda u^{\prime}\right)^{\prime}=0 \quad \text { in } \quad\right] 0,1[, \\
\lambda u^{\prime}(0)=f_{e}<0,
\end{aligned}
$$

Note that moreover, $\frac{u-u_{0}}{h}+E \geq 0$ and then, one gets that:

Proposition 2.17. If $u$ is any solution to the above problem, then $\varphi: x \mapsto \lambda u^{\prime}(x)+$ Ex is a non-decreasing continuous function. Moreover, $\varphi(0)=f_{e}$ and $\varphi(1) \geq E-f_{s}$. 
In order to know a priori if the constraint on the edge $\Gamma_{s}$ is carried out by $u(1)-u_{0}(1)+h E$ or by $\lambda u^{\prime}(1)+f_{s}$, let us consider the following remark.

Proposition 2.18. If $f_{e}+f_{s} \geq E$, then condition (10) is equivalent to the Dirichlet one

$$
u(1)=u_{0}(1)-h E .
$$

Proof. Let us consider a solution $u$ to (8-9).

Then, by multiplying (8) by $v=\left(\frac{u-u_{0}}{h}+E\right)^{-}$and using the condition (9) one gets

$$
|v|^{2} \leq-\left(\lambda u^{\prime}\right)(1) v(1)
$$

On each case, $u(1) \geq u_{0}(1)-h E$, therefore $v(1)=0$ and $\frac{u-u_{0}}{h}+E \geq 0$.

Thus, thanks to equation (8), $\varphi: x \mapsto \lambda u^{\prime}(x)+E x$ is a non-decreasing continuous function and one gets

$$
0 \leq f_{e}+f_{s}-E=\varphi(0)+f_{s}-E \leq \varphi(1)-E+f_{s}=\lambda u^{\prime}(1)+f_{s},
$$

i.e. $\lambda u^{\prime}(1)+f_{s} \geq 0$ is always satisfied.

If $u$ satisfies (8-9-10):

on the one hand, if $u=u_{0}-h E$ then (11) holds obviously,

on the other hand, if $u \neq u_{0}-h E, \varphi$ has to be increasing at least in an non-empty interval and one gets

$$
0 \leq \varphi(0)+f_{s}-E<\varphi(1)-E+f_{s}=\lambda u^{\prime}(1)+f_{s} .
$$

Therefore (10) becomes: $u(1)=u_{0}(1)-h E$. At last, if $u$ is a solution to (8-9-11), (10) is obvious.

In the sequel, $f_{e}+f_{s} \geq E$ is assumed. This scenario corresponds to a basin that empties too quickly by the boundary $\Gamma_{s}$. The boundary condition is then given by the constraint and the studied time-discretized problem is: find $u \in H^{1}(0,1)$ and $\lambda \in H\left(\frac{u-u_{0}}{h}+E\right) \cap L^{\infty}(0,1)$ such that

$$
\left.u-u_{0}-h\left(\lambda u^{\prime}\right)^{\prime}=0 \text { in }\right] 0,1\left[, \quad \lambda u^{\prime}(0)=f_{e}<0 \quad \text { and } \quad u(1)=u_{0}(1)-h E .\right.
$$

Moreover, one would consider initial conditions that satisfy:

$$
\psi: x \mapsto u_{0}(x)+\frac{E}{2} x^{2} \quad \text { is a convex function. }
$$

Note that this hypothesis is rather natural since if $\lambda=1$ in $] a, b\left[\right.$, the constraint $\frac{u-u_{0}}{h}+E \geq 0$ implies that $x \mapsto u(x)+\frac{E}{2} x^{2}$ is a convex function in $[a, b]$. Moreover, $u_{0}^{\prime} \in B V(0,1)$ and one denotes by: $u_{0}^{\prime}(0)=u_{0}^{\prime}\left(0^{+}\right)$, $u_{0}^{\prime}(1)=u_{0}^{\prime}\left(1^{-}\right)$and one assumes that for any $x, u_{0}^{\prime}(x)=\frac{u_{0}^{\prime}\left(x^{+}\right)+u_{0}^{\prime}\left(x^{-}\right)}{2}$.

One considers these assumptions mainly to be able to construct easily explicit solutions to problem (8-9-11) and confront them with the numerical results obtained in the same framework and presented in Section 2.5.

Then, numerical results of problem (8-9-10) would be presented in a more general framework.

First of all, let us consider the case when $\left(\lambda, u_{0}-h E\right)$ is a solution with $\lambda \in[0,1]$.

\subsubsection{If a trivial solution exists}

One assumes in this section that there exists $\bar{\lambda}$ in $[0,1]$ such that $\left(\bar{\lambda}, u_{0}-h E\right)$ is a solution to problem (12). 
Lemma 2.19. If $f_{e}=0$ and $E=0$, the trivial couple $\left(\bar{\lambda}=0, u_{0}\right)$ is always a solution. If $f_{e} \neq 0$ or $E \neq 0$, then $\bar{\lambda}>0$ and $u_{0}^{\prime}(x)=\frac{f_{e}-E x}{\bar{\lambda}}$.

Proof. The result comes from the fact that $\bar{\lambda} u_{0}^{\prime}(x)=f_{e}-E x$ since $\left(\bar{\lambda} u_{0}^{\prime}\right)^{\prime}=-E$ with $\bar{\lambda} u_{0}^{\prime}(0)=f_{e}$.

First case $f_{e}=0$ and $E=0$.

Proposition 2.20. Assume that $f_{e}=0$ and $E=0$, then:

(i) the trivial couple $\left(0, u_{0}\right)$ is always a solution;

(ii) if $u_{0}^{\prime}(0) \geq 0$, then a solution to (12) is given by the couple $(1, w)$ where $w$ is the solution to the problem

$$
\left.w-u_{0}-h w^{\prime \prime}=0 \quad \text { in }\right] 0,1\left[\quad \text { with } \quad w^{\prime}(0)=0 \quad \text { and } \quad w(1)=u_{0}(1)\right.
$$

(iii) if $u_{0}^{\prime}(0)<0$ and $u_{0}^{\prime}(1) \leq 0$, for any solution $(\lambda, u)$ one has $u=u_{0}$ and $0 \leq \lambda \leq 1_{\left\{u_{0}^{\prime}=0\right\}}$;

(iv) if $u_{0}^{\prime}(0)<0$ and $u_{0}^{\prime}(1)>0$, there exists $x_{0}$ in $] 0,1[$ such that a solution to (12) is given by

$$
\left.u=u_{0} \quad \text { with } \quad \lambda=0 \quad \text { in }\right] 0, x_{0}[\text { and } \quad u=w \quad \text { with } \quad \lambda=1 \quad \text { in }] x_{0}, 1[
$$

where $w$ is the solution to: $w-u_{0}-h w^{\prime \prime}=0$ in $] x_{0}, 1\left[, \quad w^{\prime}\left(x_{0}\right)=0, \quad w\left(x_{0}\right)=u_{0}\left(x_{0}\right) \quad\right.$ and $\quad w(1)=u_{0}(1)$.

Proof. As (i) is obvious, let us start by:

Claim 1. $u_{0}^{\prime}(0) \geq 0$.

Since $E=0, u_{0}$ is assumed to be a convex function, thus this condition and the maximum principle lead to $w \geq u_{0}$. Conclusion: the solution given by $\lambda=1$ is compatible with the constraint.

Claim 2. $u_{0}^{\prime}(0)<0$.

. If $u_{0}^{\prime}(1) \leq 0$. For any non-trivial solution $u$, one gets: $0=\varphi(0)<\varphi(1)=\lambda u^{\prime}(1)$.

Since $u \geq u_{0}$ and $u(1)=u_{0}(1)$, one notes that $u^{\prime}(1) \leq u_{0}^{\prime}(1)$ and a contradiction is obtained.

Thus, the trivial solution (i.e. $u=u_{0}$ ) is the unique solution to the problem.

. If $u_{0}^{\prime}(1)>0$. Let us consider $\alpha$ in ]0, 1 [ such that $u_{0}^{\prime}(\alpha) \geq 0$ and $u_{0}^{\prime}(x)<0$ if $x<\alpha$.

Then, $w$ is given by $w(x)=u_{0}(x)-\int_{x_{0}}^{x} u_{0}^{\prime}(y) \operatorname{ch}\left(\frac{y-x}{\sqrt{h}}\right) \mathrm{d} y$, where $x_{0}$ is the unique point in $] 0, \alpha[$, defined by: $\int_{x_{0}}^{1} u_{0}^{\prime}(y) \operatorname{ch}\left(\frac{y-1}{\sqrt{h}}\right) \mathrm{d} y=0$ (see Antontsev et al. [1] for some technical details).

Remark 2.21. A priori, $\mathrm{w}$ is the solution to an ill-posed elliptic problem since three boundary conditions are considered. This objection disappears since the free boundary $x_{0}$ is then characterised by this third condition.

Second case $f_{e}<0$ or $E>0$.

Proposition 2.22. In that case, only the trivial solution (i.e. $u=u_{0}-h E$ ) exists.

Proof. Let us consider a non-trivial solution $(\lambda, u)\left(i . e . u \neq u_{0}-h E\right)$. Since these solutions are continuous functions, two real numbers $a$ and $b$ exist verifying $0 \leq a<b \leq 1$ and $\left.\left\{x / u>u_{0}-h E\right\} \supset\right] a, b[$.

Since $\varphi$ is an increasing function in $] a, b[, \lambda=1$ in $] a, b\left[\right.$ and one gets that $f_{e}=\varphi(0)<\varphi(b)=u^{\prime}(b)+b E$. Moreover, since $u(1)=u_{0}(1)-h E$, one has $u(b)=u_{0}(b)-h E$ and $u \geq u_{0}-h E$. Thus, $u^{\prime}(b) \leq u_{0}^{\prime}(b)$ and

$$
f_{e}<u_{0}^{\prime}(b)+b E=\frac{f_{e}-b E}{\bar{\lambda}(b)}+b E, \quad \text { so } \quad f_{e}\left(1-\frac{1}{\bar{\lambda}(b)}\right)<E\left(1-\frac{1}{\bar{\lambda}(b)}\right),
$$

which is not possible since $\bar{\lambda}(b) \in] 0,1], f_{e}<0$ and $E>0$.

\subsubsection{Non-trivial solutions}

Remark that if $u_{0}^{\prime}(1)+E \leq f_{e}$, thanks to the convex hypothesis (13), for any $x$ in $] 0,1\left[, u_{0}^{\prime}(x)+E x \leq f_{e}<0\right.$ and $\bar{\lambda}(x):=\frac{f_{e}-E x}{u_{0}^{\prime}(x)} \in[0,1]$. Therefore, $\left(\bar{\lambda}, u_{0}-E h\right)$ is a solution and one finds again the case of the previous section. The reader is invited to look at Figure 1 for an illustration of this remark. 
Thus, let us consider in this section that $\exists \bar{b} \in[0,1], u_{0}^{\prime}(\bar{b})+\bar{b} E>f_{e}$, in particular, thanks to the hypothesis on $\psi$, for any $b$ greater than $\bar{b}$ one has the same inequality.

First case.

Proposition 2.23. If $u_{0}^{\prime}(0) \geq f_{e}$ then a solution to (12) exists with $\lambda=1$.

Proof. In that case, the maximum principle proves that the solution given by $\lambda=1$ is compatible with the constraint and of course it is the maximal one in the sense of Remark 2.3.

Remark 2.24. The reader is invited to see Figure 5 for an illustration of this case.

Second case. In that case, i.e. $u_{0}^{\prime}(0)<f_{e}$, finding a solution $(\lambda, u)$ with $\lambda=1$ on $[0,1]$ is irrelevant. Such a remark leads from the following lemma.

Lemma 2.25. No solution exists such that $\lambda=1$ in an interval $] 0, \varepsilon[$ for any given $\varepsilon>0$.

Proof. Let us assume that there exists a solution $(\lambda, u)$ such that $\lambda=1$ in $] 0, x_{0}\left[\right.$ for a given $x_{0}$ in $\left.] 0,1\right]$ such that $u\left(x_{0}\right)=u_{0}\left(x_{0}\right)-h E$. Therefore, for any $x$ in $] 0, x_{0}[$,

$$
u(x)=A \operatorname{ch}\left(\frac{x}{\sqrt{h}}\right)+B \operatorname{sh}\left(\frac{x}{\sqrt{h}}\right)-u_{0}(x)-\int_{0}^{x} \operatorname{ch}\left(\frac{y-x}{\sqrt{h}}\right) u_{0}^{\prime}(y) \mathrm{d} y
$$

where: $\quad A=-f_{e} \sqrt{h} \operatorname{th}\left(\frac{x_{0}}{\sqrt{h}}\right)+\left[\operatorname{ch}\left(\frac{x_{0}}{\sqrt{h}}\right)\right]^{-1} \int_{0}^{x_{0}} \operatorname{ch}\left(\frac{y-x_{0}}{\sqrt{h}}\right) u_{0}^{\prime}(y) \mathrm{d} y \quad$ and $\quad B=-f_{e} \sqrt{h}$.

Then, it can be proved (see Antontsev et al. [1] for technical details), for $h$ small enough, that $\frac{u-u_{0}}{h}+E<0$ on an interval $[0, \varepsilon]$ and a contradiction is found.

Thus, according to this result, let us look for a solution such that $\lambda=1$ on an interval of the form $[a, 1]$.

Proposition 2.26. For a suitable a in $] 0,1[$, a solution $w$ to the problem

$$
\left.w-h w^{\prime \prime}=u_{0} \text { on }\right] a, 1\left[\quad \text { with } \quad w(a)=u_{0}(a)-h E, \quad w(1)=u_{0}(1)-h E \quad \text { and } \quad w^{\prime}(a)+E a=f_{e},\right.
$$

exists with the additional property for any $x$ in $] 0, a\left[: \lambda(x):=\frac{f_{e}-E x}{u_{0}^{\prime}(x)} \in[0,1]\right.$.

In particular, a solution to the problem (12) exists.

Proof. Let us consider $w(x)=A \operatorname{ch}\left(\frac{x}{\sqrt{h}}\right)+B \operatorname{sh}\left(\frac{x}{\sqrt{h}}\right)-u_{0}(x)-\int_{a}^{x} \operatorname{ch}\left(\frac{y-x}{\sqrt{h}}\right) u_{0}^{\prime}(y) \mathrm{d} y$, so that $A$ and $B$ provide the solution to the Dirichlet problem:

$$
\left.w-h w^{\prime \prime}=u_{0} \text { on }\right] a, 1\left[\quad \text { with } \quad w(a)=u_{0}(a)-h E \quad \text { and } \quad w(1)=u_{0}(1)-h E .\right.
$$

Since $u_{0}^{\prime \prime}+E$ is a non-negative measure and $u_{0}^{\prime}(1)+E>f_{e}, a$ in $] 0,1\left[\right.$ exists such that $w^{\prime}(a)+E a=f_{e}$ (see Antontsev et al. [1]) and the Neumann boundary condition is satisfied.

For the additional property, note that $w^{\prime}(a) \geq u_{0}^{\prime}(a)$ with $w^{\prime}(a)+E a=f_{e}$. So $u_{0}^{\prime}(a)+E a \leq f_{e}<0$ and, as $x \mapsto u_{0}^{\prime}(x)+E x$ is an non-decreasing function, for $x \leq a$, one has $u_{0}^{\prime}(x)+E x \leq f_{e}$, i.e. $u_{0}^{\prime}(x) \leq f_{e}-E x<0$. And, for the last part of the proposition, note that by construction, this solution is $(\lambda, u)$ such that

$$
\left.\lambda=\frac{f_{e}-E x}{u_{0}^{\prime}(x)} \quad \text { with } \quad u=u_{0}-h E \quad \text { in }\right] 0, a[\quad \text { and } \quad \lambda=1 \quad \text { with } \quad \lambda=w \quad \text { else. }
$$

Remark 2.27. The previous result is a way to construct a maximal solution in the sense of Remark 2.3. But, in some particular cases, one may find in Antontsev et al. [1] a way to construct several solutions to problem (12). 
One has been able to build a non-trivial solution $u^{1}$ with $\lambda^{1}=1$ in $] a_{1}, 1\left[\right.$ where $a_{1}$ is characterized by the condition $u^{\prime}\left(a_{1}\right)+E a_{1}=f_{e}$. Note that $u^{\prime}\left(a_{1}\right) \geq u_{0}^{\prime}\left(a_{1}\right)$ and thus $u_{0}^{\prime}\left(a_{1}\right)+E a_{1} \leq f_{e}$.

Therefore, in the construction of $\left(\lambda^{2}, u_{2}\right)$ in the same way, $a_{2}$ can be chosen less then $a_{1}$. By induction, a non-increasing sequence $\left(a_{k}\right)_{k}$ may be constructed such that:

$$
\lambda^{k}(x)=\mu^{k} 1_{\left[0, a_{k}[\right.}+1_{] a_{k}, 1\right]} \quad \text { and } \quad u^{k}=\left(u_{0}-k h E\right) 1_{] 0, a_{k}\right]}+w^{k} 1_{] a_{k}, 1\right]} \quad \text { where } \quad \mu^{k}=\frac{f_{e}-E x}{u_{k-1}^{\prime}(x)}
$$

and $w^{k}$ is the solution to:

$$
\left.\left.w^{k}-h\left(w^{k}\right)^{\prime \prime}=u_{0} \text { in }\right] a_{k}, 1\right] \quad \text { with } \quad w^{k}\left(a_{k}\right)=u_{0}\left(a_{k}\right)-k h E, \quad w^{k}(1)=u_{0}(1)-k h E \quad \text { and } \quad\left(w^{k}\right)^{\prime}\left(a_{k}\right)=f_{e}
$$

Therefore, considering the notation of Property 2.15, $\lambda_{h}=1_{\omega_{h}}+\lambda_{h}\left(1-1_{\omega_{h}}\right)$ where $\omega_{h}$ is a set of uniform bounded perimeter. Thus, passing to the limits is possible in the formulation, for any $v$ in $H^{1}(0,1)$ with $v(1)=0$

$$
0=\int_{\Omega}\left\{\partial_{t} \hat{u}_{h} v+\lambda_{h} \nabla u_{h} . \nabla v\right\} \mathrm{d} x+\int_{\Gamma_{e}} f_{e} v \mathrm{~d} \sigma
$$

since $u_{h}$ is known in the set where $\lambda_{h}$ is not equal to 1 (see Gagneux et al. [10]).

Remark 2.28. The reader is invited to consult the simulations given in Figures 2-4.

\section{5. $1-D$ numerical simulations}

Obviously, even in the $1-D$ case, the numerical discretization of the equation defined by (1) to (5) presents lots of difficulties. The most important are the calculus of $\lambda$ and the unilateral constraint. We present a relatively simple algorithm which allows us to illustrate some theoretical results and to present some interesting simulations.

In order to solve the equation defined by (1) to (5), we discretize the time derivative by using an implicit Euler scheme and the space derivative by using a $\mathrm{P}_{1}$-conform finite element method. The function $\lambda$ is approached by constants by piece.

Define $[0,1]=\cup_{i=0}^{n-1}\left[x_{i}, x_{i+1}\right], W$ the space of hat-functions and $u^{k}(x)$ an approximation of $u(k h, x)$ in $W$.

The variational formulation of the semi-discretized problem is the following:

$$
\mid \begin{aligned}
& \text { find } u^{k+1} \text { in } W \text { satisfying }, \forall v_{j} \in W \subset H^{1}(0,1) \\
& \int_{\Omega} \frac{u^{k+1}-u^{k}}{h} v_{j} \mathrm{~d} x+\int_{\Omega} \lambda\left(u^{k+1}, u^{k}\right) u^{k+1^{\prime}} v_{j}^{\prime} \mathrm{d} x+f_{e} v_{j}(0)=0 \\
& \lambda u^{k+1^{\prime}}(0)=f_{e}, \\
& \lambda u^{k+1^{\prime}}(1)+f_{s} \geq 0_{e}, u^{k+1}(1)-u^{k}(1)+h E \geq 0 \text { and }\left(\lambda u^{k+1 \prime}(1)+f_{s}\right)\left(u^{k+1}(1)-u^{k}(1)+h E\right)=0 .
\end{aligned}
$$

At each time step, a non-linear equation must be solve and a fixed-point algorithm is used. The base of this algorithm is to determine the value of $\lambda$ and the boundary condition at $x=1$ from the value of $u$ at the previous iteration.

$$
\mid \begin{aligned}
& \text { For } l=1,2, \ldots, \forall v_{j} \in W, \\
& \int_{\Omega} \frac{u^{k+1, l+1}-u^{k}}{h} v_{j} \mathrm{~d} x+\int_{\Omega} \lambda\left(u^{k+1, l}, u^{k}\right) u^{k+1, l+1^{\prime}} v_{j}^{\prime} \mathrm{d} x+f_{e} v_{j}(0)=0, \\
& + \text { boundary conditions, } \\
& \text { with } u^{k+1,0}=u^{k} .
\end{aligned}
$$




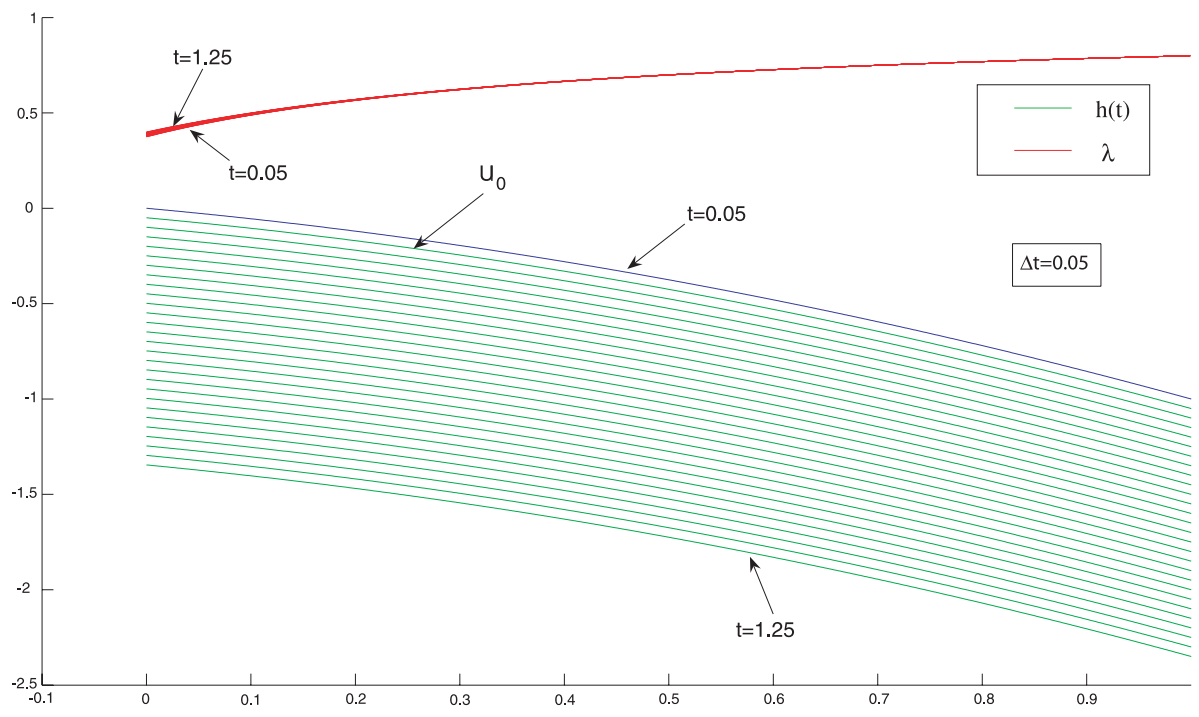

Figure 1. $E=1, f_{e}=-0.2$ and $u_{0}^{\prime}(1)=-1.5$.

Supposed given $\lambda\left(u^{k+1, l}, u^{k}\right)$ and the boundary condition at $x=1$, then $u^{k+1, l+1}$ is solution of a linear system and the process is stopped when the series converges.

Let us denote the discrete operators:

$$
D_{t} u_{j}=\frac{u_{j}^{k+1, l}-u_{j}^{k}}{h}, \quad D_{x, 0} u_{j}=\frac{u_{j}^{k}-u_{j-1}^{k}}{x_{j}-x_{j-1}}, \quad D_{x, 1} u_{j}=\frac{u_{j}^{k+1, l}-u_{j+1}^{k+1, l}}{x_{j}-x_{j-1}}
$$

To construct $\lambda\left(u^{k+1, l}, u^{k}\right)$, Proposition 2.17 is taken into account (continuity of $\varphi$ ). So we have the following algorithm:

- $\lambda_{j}=0(1 \leq j \leq n)$

- if $D_{t} u_{j-1}+E>0$ and $D_{t} u_{j}+E>0$ then $\lambda_{j}=1$. We denote by $I=\cup_{i=1}^{m}\left[x_{p_{i}^{1}}, x_{p_{i}^{2}}\right]$ the set where $\lambda=0$.

- for $j \in\left[p_{1}^{1}, p_{1}^{2}\right], \lambda_{j}=\frac{f_{e}+E x_{j}}{D_{x, 0} u_{j}}$

- for $i \in[2, m]$, for $j \in\left[p_{i}^{1}, p_{i}^{2}\right] \lambda_{j}=\frac{D_{x, 1} u_{p_{i}^{1}}+E x_{j}}{D_{x, 0} u_{j}}$.

- if $\lambda_{j}>1$ then $\lambda_{j}=1$.

At each step of the process, the unilateral constraint at $x=1$ can give either a Dirichlet or a Neumann condition. To determine it, we propose the following simple algorithm:

- if the boundary condition is of Dirichlet type at step $k+1, l$ and $\lambda\left(u^{k+1, l}, u^{k}\right) D_{x, 0} u_{n}+f_{s}<0$, then at step $k+1, l+1$, we impose $\lambda\left(u^{k+1, l}, u^{k}\right) u^{k+1, l+1 \prime}(1)+f_{s}=0$.

- if the boundary condition is of Neumann type at step $k+1, l$ and $u^{k+1, l}(1)-u^{k}(1)+h E<0$, then at step $k+1, l+1$, we impose $u^{k+1, l+1}(1)-u^{k}(1)+h E=0$.

Then, the space of discretization $W$ is modified to take into account the type of boundary condition. 


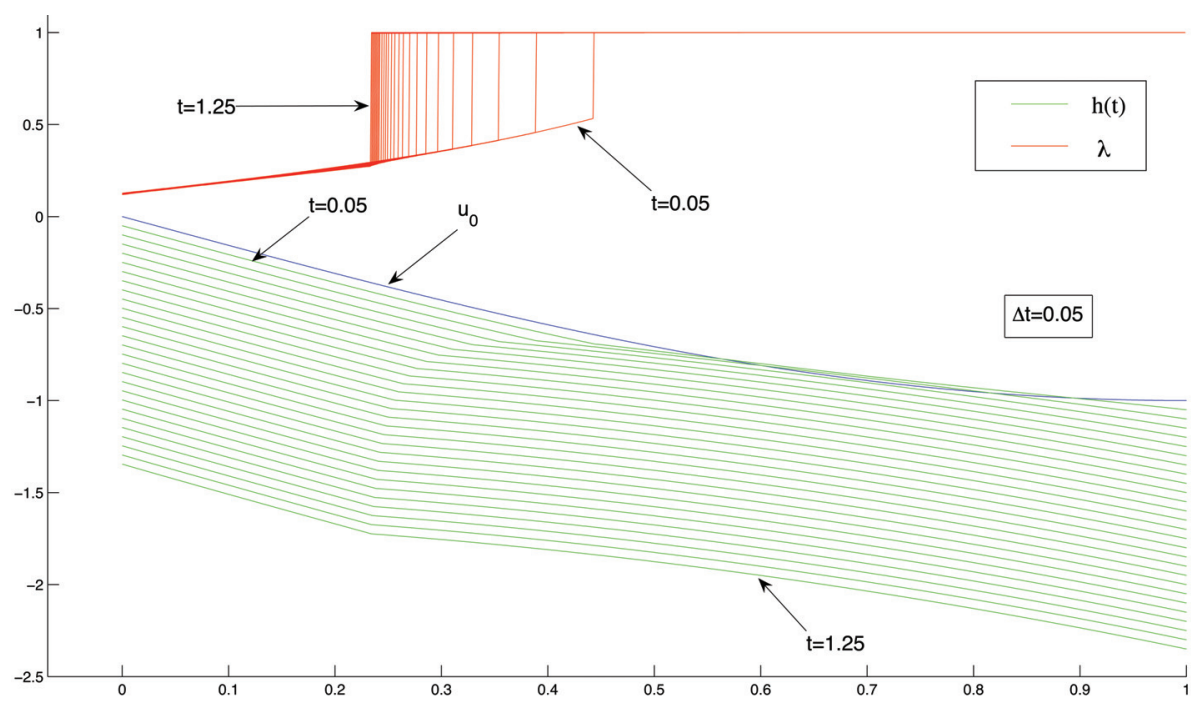

Figure 2. $E=1, f_{e}=-0.2$ and $u_{0}^{\prime}(0)=-\frac{\pi}{2}$.

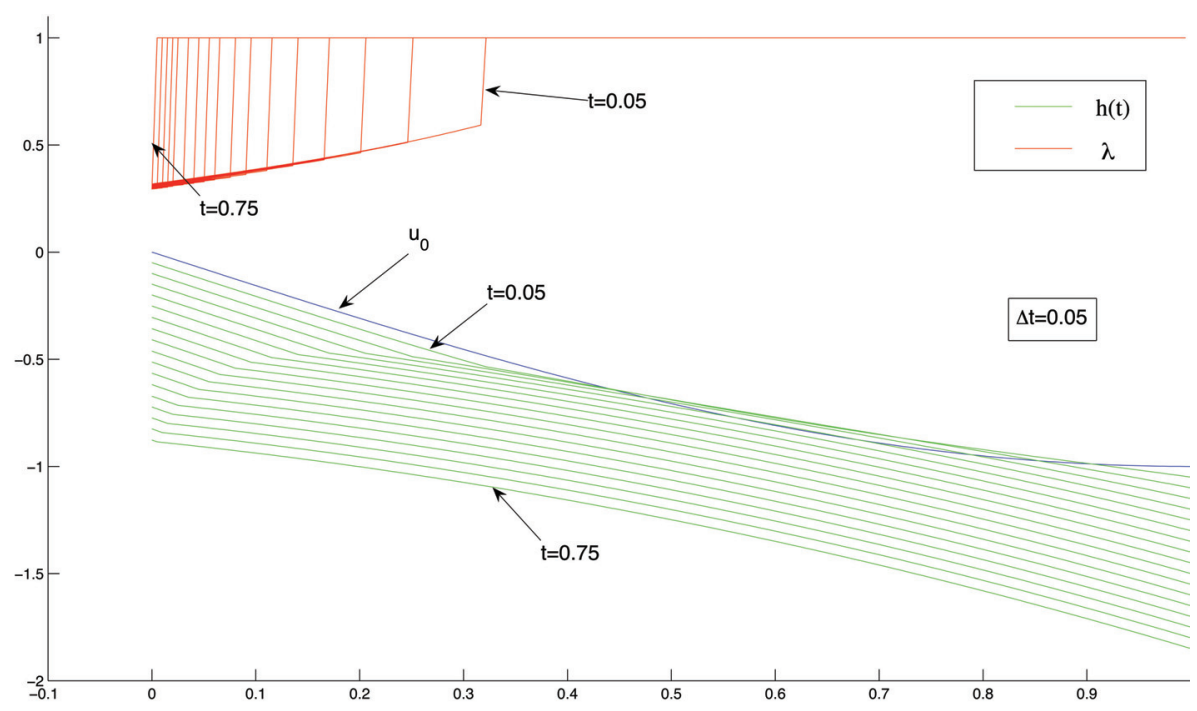

Figure 3. $E=1, f_{e}=-0.5$ and $u_{0}^{\prime}(0)=-\frac{\pi}{2}$.

\section{Remark 2.29.}

- There are no theoretical results about the convergence of the fixed-point.

- At each step of the fixed-point, the finite element matrix must be assembled again. If an explicit time scheme is used, the fixed-point does not converge.

In Figures 1 to 5 , one presents numerical simulations of phenomena described in Section 2.4 when $f_{e}+f_{s} \geq E$. As expected, at each iteration, the numerical process chooses the Dirichlet boundary condition at $x=1$.

Figures 6 to 11 present numerical tests obtained for two initial conditions $u_{0}$ and different values of $\left(E, f_{e}, f_{s}\right)$. With these values, the unilateral boundary condition is not predetermined as previously. Then, the numerical 


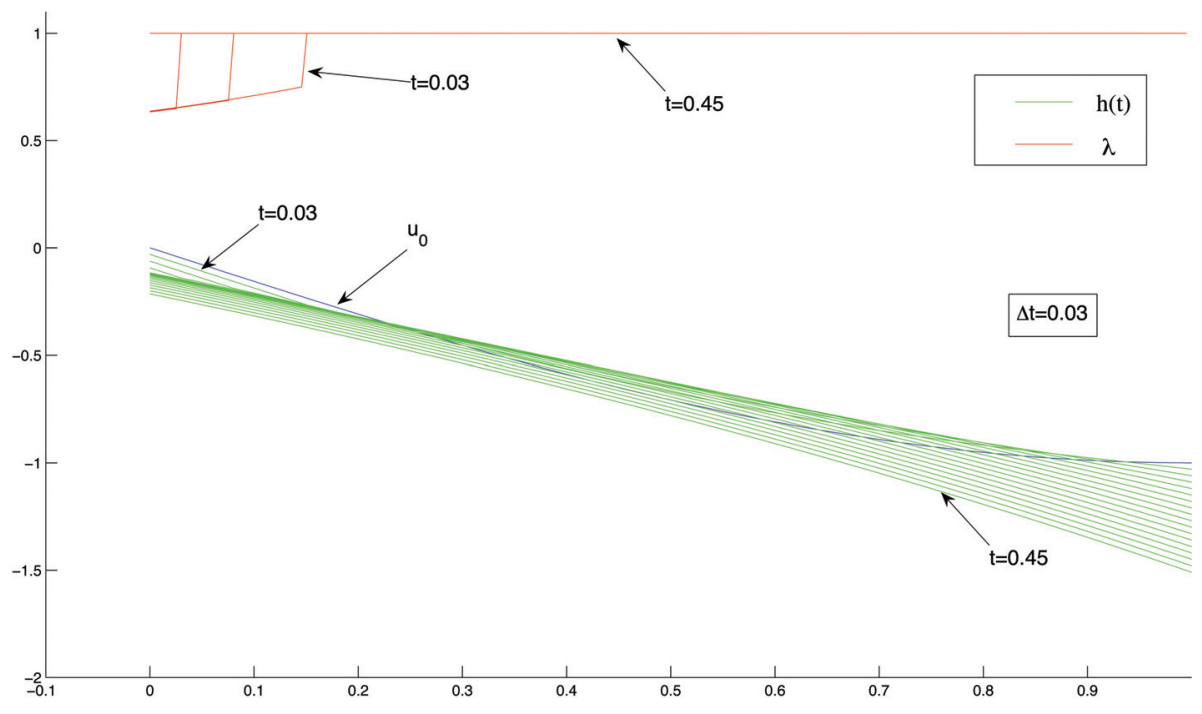

Figure $4 . E=1, f_{e}=-1$ and $u_{0}^{\prime}(0)=-\frac{\pi}{2}$.

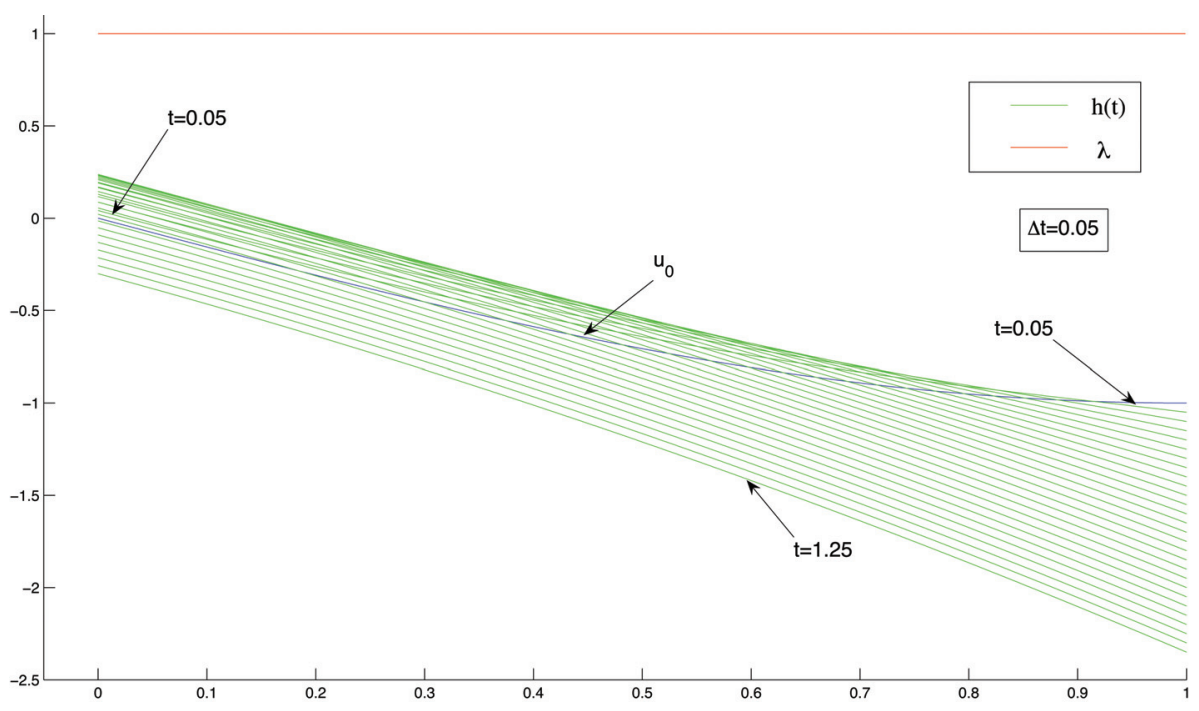

Figure 5. $E=1, f_{e}=-1.6$ and $u_{0}^{\prime}(0)=-\frac{\pi}{2}$.

process has to select the right one. In the first example (Figs. 6 to 8 ), $u_{0}$ is decreasing. If $f_{e}+f_{s}<E$, the algorithm imposes the Neumann condition (Figs. 6, 7) and the Dirichlet condition else (Fig. 8).

In the second example (Figs. 9 to 11), the initial condition $u_{0}$ in not a monotone function and one observes that the boundary type has to change after a time when $f_{e}+f_{s}<E$ (Figs. 9, 10) ${ }^{1}$. In the other case, as expected in Section 2.4, only the Dirichlet condition occurs (Fig. 11).

\footnotetext{
${ }^{1}$ A single line if the Neumann-condition is chosen, a dash line else.
} 


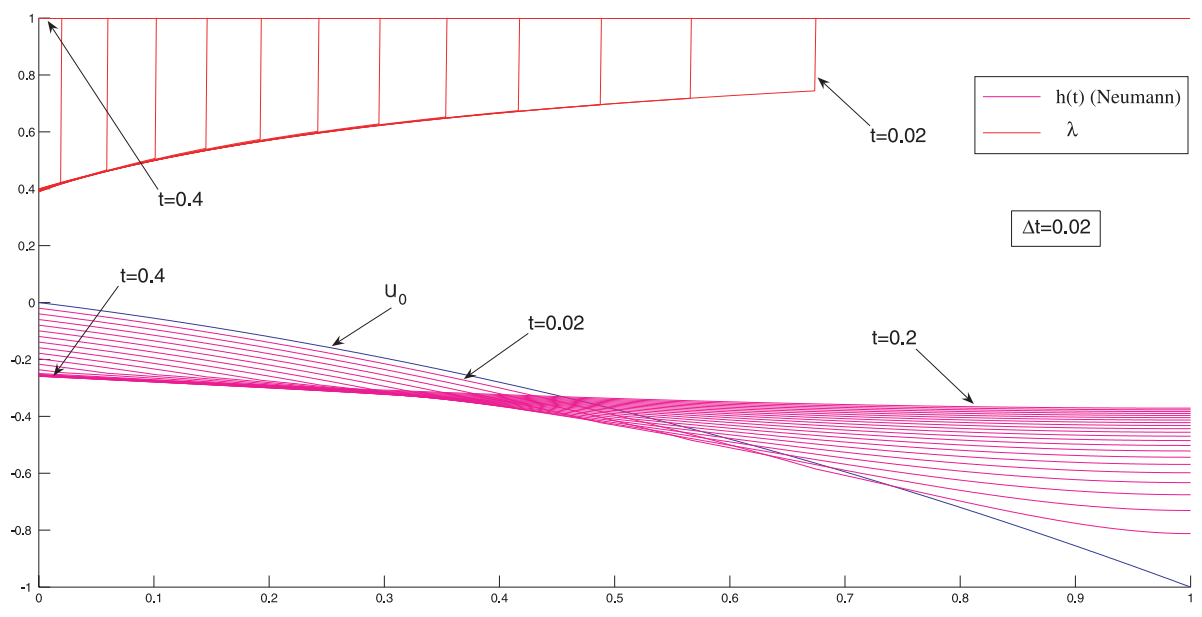

FiguRE 6. $E=1, f_{e}=-0.2$ and $f_{s}=0$.

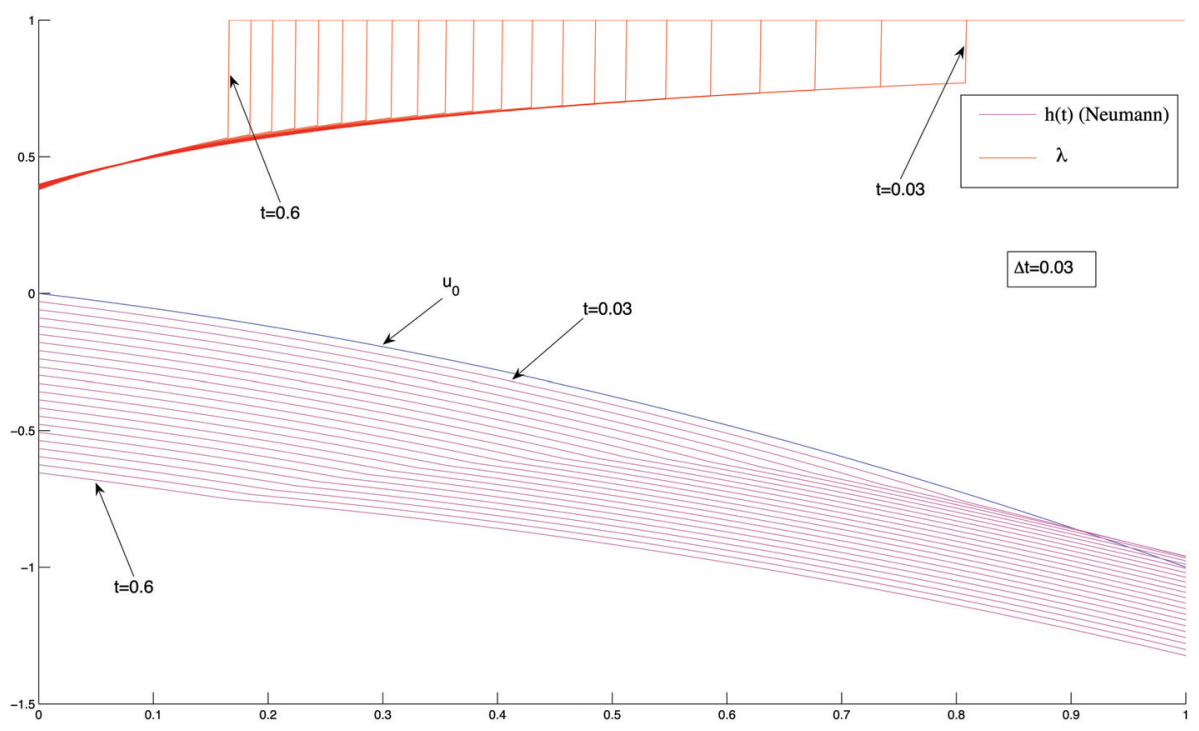

Figure 7. $E=1, f_{e}=-0.2$ and $f_{s}=1$.

All figures seem to be in agreement with the phenomena of transport and erosion modelled by the equation and the unilateral constraint.

\subsection{Conclusion and open problem}

One has been able to extend the results already obtained in the case of homogeneous Dirichlet boundary conditions. This study concerns a more acceptable physically problem. The boundary conditions are of unilateral type and lead to a variational inequality, in order to satisfy the maximal erosion constraint.

Of course, the existence of a solution is still an open problem, mainly since one needs more information on the convergence of the term $\int_{\Omega} \lambda_{h} \nabla u_{h} . \nabla v \mathrm{~d} x$. Nevertheless, in the $1-D$ case, as presented in Vallet [18] and 


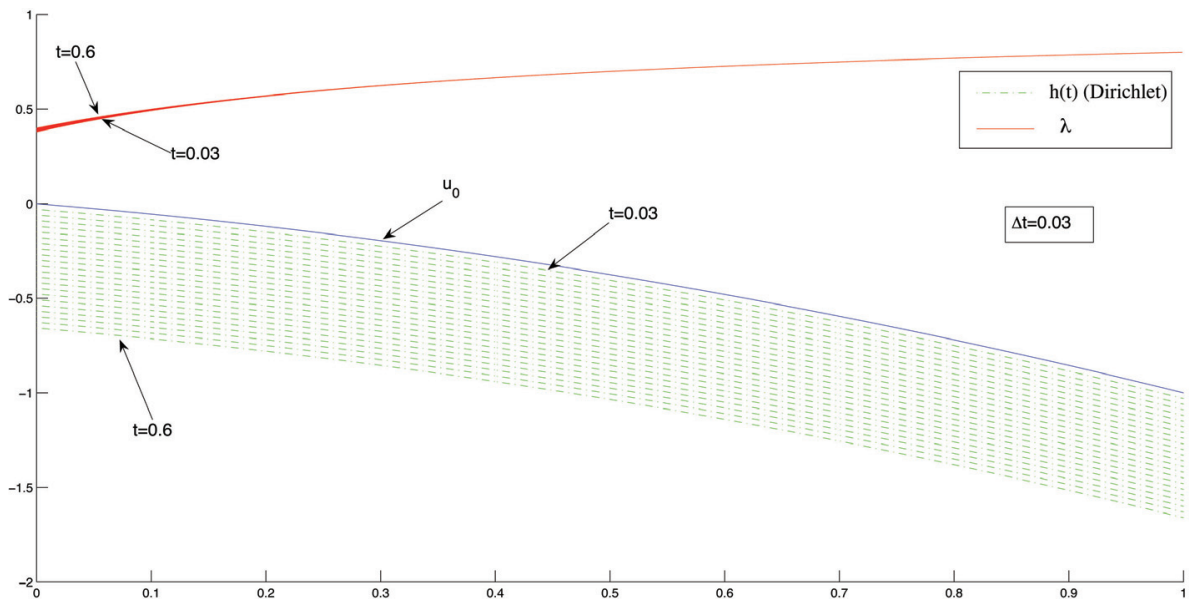

Figure 8. $E=1, f_{e}=-0.2$ and $f_{s}=1.8$.

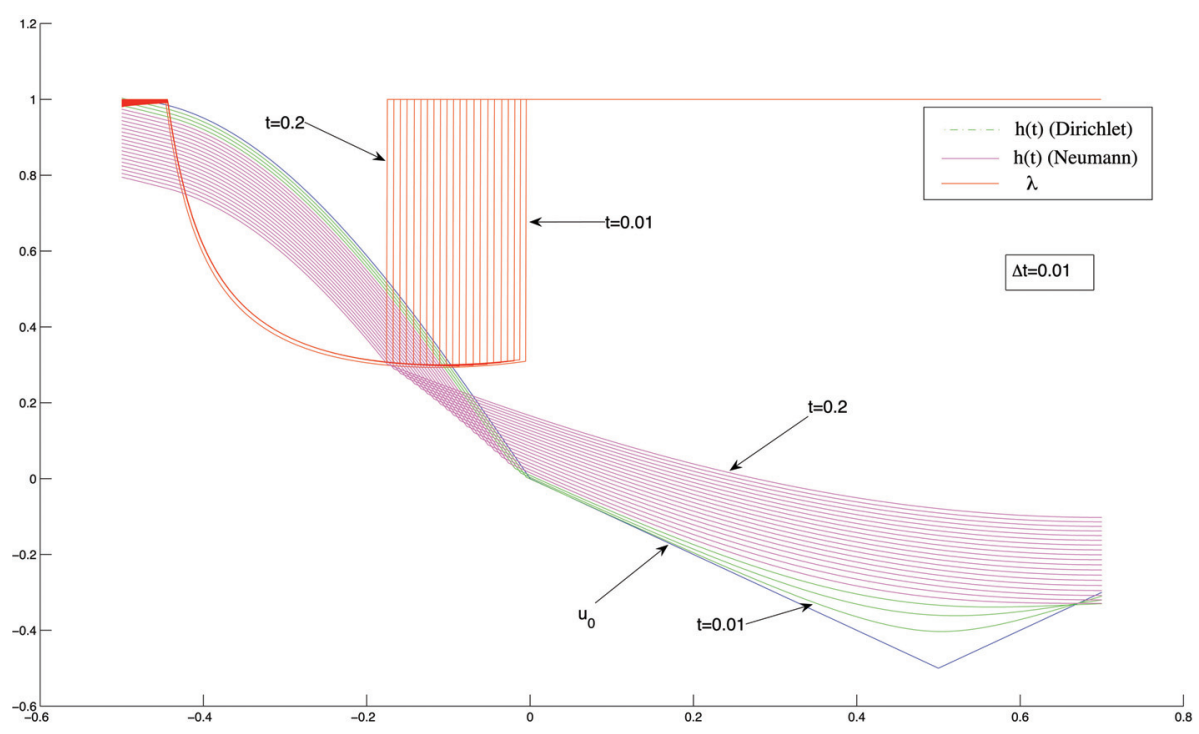

Figure $9 . \quad E=1, f_{e}=-0.5$ and $f_{s}=0$.

Gagneux et al. [10] for the Dirichlet problem, it has been possible to get rid of the difficulty by an approach based on the properties of bounded variation sets.

An other difficulty appears since in the formulation and in the boundary condition on $\Gamma_{s}$, a priori estimates in $L^{2}\left(0, T ; H^{1}(\Omega)\right)$ for $\partial_{t} \hat{u}_{h}$ is explicitly needed. Therefore, one has to consider a generalization of the Darcy's law in the following sense: $\vec{q}=-\lambda \nabla\left[u+\tau \partial_{t} u\right]$ in $Q$ where $\tau>0$, according to the Barenblatt model in the non-static situation, see Cuesta et al. [6]. One proposes a few words about that in Gagneux et al. [13] and Antontsev et al. [2].

At last, one has to think about a robust numerical method in order to simulate such non-linear models. The main problem is to consider a fixed-point method with multi-valued applications. 


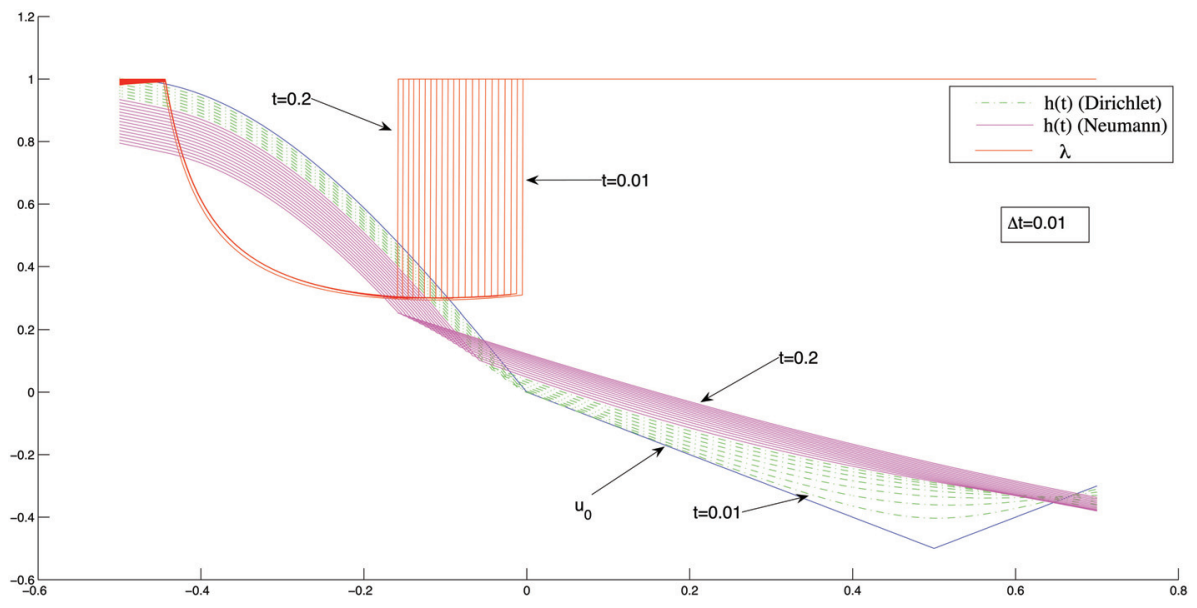

Figure 10. $E=1, f_{e}=-0.5$ and $f_{s}=0.5$.

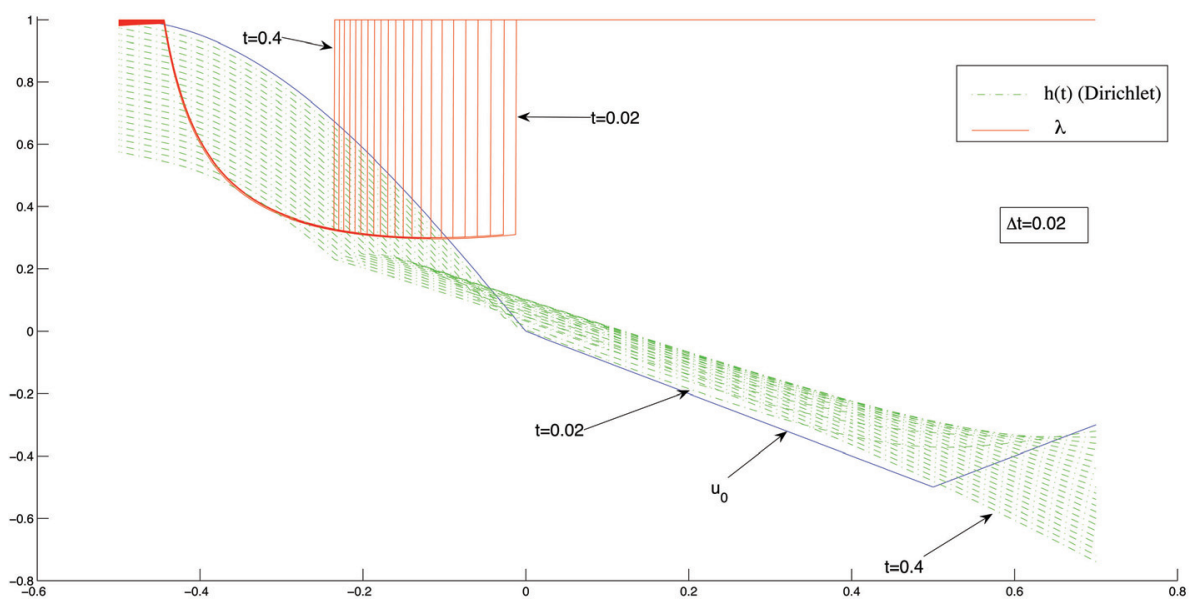

Figure 11. $E=1, f_{e}=-0.5$ and $f_{s}=2$.

\section{REFERENCES}

[1] S.N. Antontsev, D. Etienne, G. Gagneux and G. Vallet, New unilateral problems in stratigraphy. Publication interne du Laboratoire de Mathématiques Appliquées, UMR-CNRS 5142, Université de Pau, No. 05/13 (2005).

[2] S.N. Antontsev, G. Gagneux, R. Luce and G. Vallet, Weather limited constraint in stratigraphy. International conference "Tikhonov and Contemporary Mathematics": section 5, Mathematical Geophysics, Moscow (2006) 7-8.

[3] S.N. Antontsev, G. Gagneux and G. Vallet, Analyse mathématique d'un modèle d'asservissement stratigraphique. Approche gravitationnelle d'un processus de sédimentation sous contrainte d'érosion maximale. Publication interne du Laboratoire de Mathématiques Appliquées, UMR-CNRS 5142, Université de Pau, No. 01/23 (2001).

[4] S.N. Antontsev, G. Gagneux and G. Vallet, On some stratigraphic control problems, Prikladnaya Mekhanika Tekhnicheskaja Fisika (Novosibirsk) 44 (2003) 85-94 (in Russian), and Journal of Applied Mechanics and Technical Physics (New York) 44 (2003) 821-828.

[5] P. Bénilan, M.G. Crandall and A. Pazy, Bonnes solutions d'un problème d'évolution semi-linéaire. C. R. Acad. Sci. Paris Sér. I Math. (306) (1988) 527-530.

[6] C. Cuesta, C.J. van Duijn and J. Hulshof, Infiltration in porous media with dynamic capillary pressure: Travelling waves. Eur. J. Appl. Math. 11 (2000) 381-397. 
[7] D. Etienne, Contribution à l'analyse mathématique de modèles stratigraphiques. Thèse de l'Université de Pau, France (2004).

[8] R. Eymard and T. Gallouët, Analytical and numerical study of a model of erosion and sedimentation. SIAM J. Numer. Anal. 43 (2006) 2344-2370.

[9] R. Eymard, T. Gallouët, D. Granjeon, R. Masson and Q.H. Tran, Multi-lithology stratigraphic model under maximum erosion rate constraint. Internat. J. Numer. Methods Engrg. 60 (2004) 527-548.

[10] G. Gagneux, R. Luce and G. Vallet, A non-standard free-boundary problem arising from stratigraphy. Publication interne du Laboratoire de Mathématiques Appliquées, UMR-CNRS 5142, Université de Pau, No. 04/33 (2004).

[11] G. Gagneux and M. Madaune-Tort, Analyse mathématique de modèles non linéaires de l'ingénierie pétrolière. Mathématiques \& Applications, Vol. 22 Springer, Paris (1996).

[12] G. Gagneux and G. Vallet, Sur des problèmes d'asservissements stratigraphiques. ESAIM: COCV "A tribute to Jacques-Louis Lions" 8 (2002) 715-739.

[13] G. Gagneux and G. Vallet, A result of existence for an original convection-diffusion equation. Revista de la Real Academia de Ciencias, Serie A: Matemáticas (RACSAM) 99 (2005) 125-131.

[14] T. Gallouët, Equations satisfaites par des limites de solutions approchées, conférence plénière, 34ème congrès d'analyse numérique, Anglet (Pyrénées Atlantiques), in Canum (2002) 87-96.

[15] V. Gervais and R. Masson, Mathematical and numerical analysis of a stratigraphic model. ESAIM: M2AN 38 (2004) 585-611.

[16] J.-L. Lions, Cours d'Analyse Numérique. Hermann, École Polytechnique, Paris (1973).

[17] J.-L. Lions, Quelques méthodes de résolution des problèmes aux limites non linéaires. Dunod, Paris (1969).

[18] G. Vallet, Sur une loi de conservation issue de la géologie. C. R. Acad. Sci. Paris Sér. I Math. (337) (2003) 559-564.

To access this journal online: www.edpsciences.org 